\title{
Mass and Rate of Hierarchical Black Hole Mergers in Young, Globular and Nuclear Star Clusters
}

\author{
Michela Mapelli 1,2,3*(D), Filippo Santoliquido ${ }^{1,2}\left(\mathbb{D}\right.$, Yann Bouffanais ${ }^{1,2} \mathbb{D}$, Manuel Arca Sedda 4 (D), \\ Maria Celeste Artale ${ }^{5}$ (D) and Alessandro Ballone ${ }^{1,2,3}$ (D)
}

1 Physics and Astronomy Department Galileo Galilei, University of Padova, Vicolo dell'Osservatorio 3, I-35122 Padova, Italy; filippo.santoliquido@phd.unipd.it (F.S.); yann.bouffanais@unipd.it (Y.B.); alessandro.ballone@unipd.it (A.B.)

2 INFN-Padova, Via Marzolo 8, I-35131 Padova, Italy

3 INAF-Osservatorio Astronomico di Padova, Vicolo dell'Osservatorio 5, I-35122 Padova, Italy

4 Astronomisches Rechen-Institut, Zentrum für Astronomie der Universität Heidelberg, Mönchhofstrasse 12-14, D-69120 Heidelberg, Germany; m.arcasedda@uni-heidelberg.de

5 Institut für Astro- und Teilchenphysik, Universität Innsbruck, Technikerstrasse 25/8,

A-6020 Innsbruck, Austria; Maria.Artale@uibk.ac.at

* Correspondence: michela.mapelli@unipd.it

check for updates

Citation: Mapelli, M.; Santoliquido, F.; Bouffanais, Y.; Arca Sedda, M.; Artale, M.C.; Ballone, A. Mass and Rate of Hierarchical Black Hole Mergers in Young, Globular and Nuclear Star Clusters. Symmetry 2021, 13, 1678. https://doi.org/ 10.3390/sym13091678

Academic Editors: José A. de Freitas Pacheco and Pasquale Bosso

Received: 17 August 2021

Accepted: 10 September 2021

Published: 12 September 2021

Publisher's Note: MDPI stays neutral with regard to jurisdictional claims in published maps and institutional affiliations.

Copyright: (c) 2021 by the authors. Licensee MDPI, Basel, Switzerland. This article is an open access article distributed under the terms and conditions of the Creative Commons Attribution (CC BY) license (https:// creativecommons.org/licenses/by/ $4.0 /)$.
Abstract: Hierarchical mergers are one of the distinctive signatures of binary black hole (BBH) formation through dynamical evolution. Here, we present a fast semi-analytic approach to simulate hierarchical mergers in nuclear star clusters (NSCs), globular clusters (GCs) and young star clusters (YSCs). Hierarchical mergers are more common in NSCs than they are in both GCs and YSCs because of the different escape velocity. The mass distribution of hierarchical BBHs strongly depends on the properties of first-generation BBHs, such as their progenitor's metallicity. In our fiducial model, we form black holes (BHs) with masses up to $\sim 10^{3} \mathrm{M}_{\odot}$ in NSCs and up to $\sim 10^{2} \mathrm{M}_{\odot}$ in both GCs and YSCs. When escape velocities in excess of $100 \mathrm{~km} \mathrm{~s}^{-1}$ are considered, BHs with mass $>10^{3} \mathrm{M}_{\odot}$ are allowed to form in NSCs. Hierarchical mergers lead to the formation of BHs in the pair instability mass gap and intermediate-mass BHs, but only in metal-poor environments. The local BBH merger rate in our models ranges from $\sim 10$ to $\sim 60 \mathrm{Gpc}^{-3} \mathrm{yr}^{-1}$; hierarchical BBHs in NSCs account for $\sim 10^{-2}-0.2 \mathrm{Gpc}^{-3} \mathrm{yr}^{-1}$, with a strong upper limit of $\sim 10 \mathrm{Gpc}^{-3} \mathrm{yr}^{-1}$. When comparing our models with the second gravitational-wave transient catalog, we find that multiple formation channels are favored to reproduce the observed $\mathrm{BBH}$ population.

Keywords: astrophysical black holes; intermediate-mass black holes; gravitational waves; star clusters

\section{Introduction}

The past six years have witnessed the first three observing runs of the Advanced LIGO and Virgo gravitational wave (GW) interferometers [1,2], leading to the detection of about 50 binary compact object mergers [3-11]. This growing sample of GW observations represents a "Rosetta stone" to investigate the formation of binary compact objects.

Several channels can lead to the formation of binary black holes (BBHs): Pairing of primordial black holes (e.g., [12-14]), binary star evolution through common envelope (e.g., [15-38]) or via homogeneous mixing (e.g., [39-42]), dynamical processes in triples (e.g., [43-47]), young/open star clusters (YSCs, e.g., [48-58]), globular clusters (GCs, e.g., [59-68]), nuclear star clusters (NSCs, e.g., [69-77]) and AGN disks (e.g., [78-83]).

One of the distinctive signatures of the dynamical scenario is the formation of hierarchical mergers, i.e., repeated mergers of stellar-origin black holes (BHs) that build up more massive ones [84-88]. This process is possible only in dense star clusters, where the merger remnant, which is initially a single $\mathrm{BH}$, can acquire a companion by dynamical exchanges [89]. The main obstacle to the formation of second-generation (2g) BHs via hierarchical mergers is the high relativistic kick that the merger remnant receives at birth 
because of radiation of linear momentum through beamed GW emission (e.g., [90-93]). This kick can be up to several thousand $\mathrm{km} \mathrm{s}^{-1}$ and can easily eject the $\mathrm{BH}$ remnant from its parent star cluster [76,94-97]. Hence, the interplay between the properties of the host star cluster (e.g., its escape velocity), those of the first-generation (1g) BBH population and the magnitude of the kick decides the maximum mass of a merger remnant in a given environment. This might be used to constrain the formation channels of BBHs.

The spins of $1 \mathrm{~g}$ BHs are one of the critical ingredients because relativistic kicks are sensitive to spin magnitudes and orientation (e.g., [98,99]). In the zero-spin assumption, more than $10 \%$ of merging BBHs from GCs have components formed from previous mergers, accounting for more than $20 \%$ of the mergers from GCs detectable by Advanced LIGO and Virgo [100].

Due to their high escape velocity $\left(v_{\mathrm{esc}} \sim 100 \mathrm{~km} \mathrm{~s}^{-1}\right)$, NSCs are more likely to retain hierarchical mergers than other star clusters (e.g., [71,76,82,101]). Antonini et al. (2019, [102]) recently found that $\mathrm{BH}$ growth becomes substantial for $v_{\mathrm{esc}}>300 \mathrm{~km} \mathrm{~s}^{-1}$, leading to the formation of intermediate-mass BHs (IMBHs, see also [47]). Hence, hierarchical mergers can build up IMBHs and also partially fill the pair instability mass gap between $\sim 60$ and $\sim 120 \mathrm{M}_{\odot}$ [103-108]. For this reason, hierarchical mergers are one of the most likely formation scenarios for GW190521 [109,110], as already explored by several authors ([111-116], but see $[55,56,117-126]$ for other possible scenarios).

The main challenge of studying hierarchical mergers is the computational cost. It is nearly impossible to investigate the relevant parameter space with hybrid Monte Carlo and/or N-body simulations of star clusters, especially GCs and NSCs. Here, we present a new fast and flexible semi-analytic model to investigate hierarchical mergers in different environments, complementary to dynamical simulations. Our new tool allows us to probe the parameter space (1g masses, spins, delay times, $2 \mathrm{~g}$ masses, spins and delay times, escape velocity from the parent cluster and kick magnitudes) and to reconstruct the merger rate evolution of each formation channel, with just minimal model assumptions.

\section{Methods}

We consider four different environments: (i) The field, where hierarchical mergers are not possible, (ii) young star clusters (YSCs), which are the main birth site of massive stars in the local Universe (e.g., [127]), (iii) globular clusters (GCs), and (iv) nuclear star clusters (NSCs). To evaluate the properties of $1 \mathrm{~g}$ mergers, we start from catalogs of single and binary $\mathrm{BHs}$ obtained with population-synthesis simulations. When the $1 \mathrm{~g}$ BHs merge, we estimate the relativistic kick $v_{\text {kick }}$ and the escape velocity from the parent star cluster $v_{\text {esc }}$. If $v_{\text {kick }}<v_{\text {esc }}$, we assume that the merger remnant remains bound to its parent star cluster and can pair with another $\mathrm{BH}$ dynamically. We estimate the mass and spin of the merger remnant and of its new companion, as detailed below. Then, we randomly draw a new delay time between previous and next merger. If the sum of the new delay time and the previous one is shorter than the Hubble time, we repeat the loop for another generation.

\subsection{First Generation (1g) Mergers}

We take the mass of $1 \mathrm{~g}$ BHs from our population synthesis simulations. In particular, we used our code MOBSE $[28,30,31]$. MOBSE is an upgraded and customized version of BSE [128]. The treatment of stellar winds is one of the key aspects affecting the final mass of BHs and is subject to a number of uncertainties [129]. In MOBSE, mass loss by stellar winds for massive host stars (O-type, B-type, luminous blue variable and Wolf-Rayet stars) is modeled as $\dot{M} \propto Z^{\beta}$, where $Z$ is the metallicity and

$$
\beta= \begin{cases}0.85, & \text { if } \Gamma_{e} \leq 2 / 3 \\ 2.45-2.4 \Gamma_{e}, & \text { if } 2 / 3<\Gamma_{e} \leq 1 \\ 0.05, & \text { if } \Gamma_{e}>1\end{cases}
$$

In Equation (1), $\Gamma_{e}$ is the Eddington ratio, i.e., the ratio between the luminosity of the star and its Eddington value. This formalism, introduced by [130], is a fit to the 
models presented in [131]. It accounts for both the $Z$ dependence of line-driven winds [132] and the importance of Thomson scattering when a star is nearly radiation pressure dominated [131,133].

In detail, for $\mathrm{O}$ and B-type stars with effective temperature $T_{\text {eff }} \geq 12,500 \mathrm{~K}$, we use the same fitting formulas as introduced by [132], but we correct the $Z$ dependence as described in Equation (1). We model the mass loss rate of Wolf-Rayet stars as [134]

$$
\dot{M}=10^{-13} L^{1.5}\left(\frac{\mathrm{Z}}{\mathrm{Z}_{\odot}}\right)^{\beta} \mathrm{M}_{\odot} \mathrm{yr}^{-1} .
$$

The mass loss rate of luminous blue variable stars is [30]

$$
\dot{M}=1.5 \times 10^{-4}\left(\frac{\mathrm{Z}}{\mathrm{Z}_{\odot}}\right)^{\beta} \mathrm{M}_{\odot} \mathrm{yr}^{-1} .
$$

Finally, the treatment of mass loss of cold massive stars is the same as originally described by [135].

The effect of core-collapse supernovae on the mass of compact objects is described following the delayed model of [136]. According to this model, stars with final carbonoxygen mass $m_{\mathrm{CO}} \gtrsim 11 \mathrm{M}_{\odot}$ collapse to a $\mathrm{BH}$ directly. The minimum $\mathrm{BH}$ mass is $3 \mathrm{M}_{\odot}$. Following $[137,138]$, we compute neutrino mass loss for both neutron stars and BHs as

$$
m_{v}=\min \left[\frac{\left(\sqrt{1+0.3 m_{\mathrm{bar}}}-1\right)}{0.15}, 0.5 \mathrm{M}_{\odot}\right]
$$

where $m_{\mathrm{bar}}$ is the baryonic mass of the compact object. The resulting gravitational mass of the compact object is $m_{\text {grav }}=m_{\text {bar }} m_{v}$.

Stars with helium core mass (at the end of carbon burning) $32 \leq m_{\mathrm{He}} \leq 64$ and $64 \leq m_{\mathrm{He}} \leq 135$ undergo pulsational pair instability and pair instability supernovae, respectively [104]. Stars that undergo a pair instability supernova leave no compact remnant, while stars going through pulsational pair instability become BHs with mass

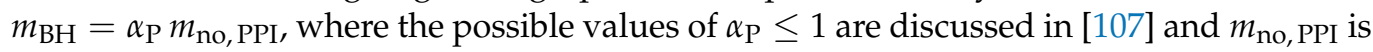
the $\mathrm{BH}$ mass from direct collapse, if pulsational pair instability is not accounted for. Finally, electron-capture supernovae are included following [139]. For natal kicks, we adopt the prescription $v_{\mathrm{k}} \propto m_{\mathrm{ej}} m_{\mathrm{rem}}^{-1}$, where $m_{\mathrm{ej}}$ is the mass of the ejecta and $m_{\text {rem }}$ is the mass of the compact remnant (neutron star or $\mathrm{BH},[140]$ ).

Binary evolution processes (wind mass transfer, Roche lobe overflow, common envelope, mergers, tidal evolution, GW decays) are implemented as in [128], with one significant exception. During Roche lobe overflow, the accretion rate is calculated as

$$
\dot{m}_{a}= \begin{cases}f_{\mathrm{MT}}\left|\dot{m}_{d}\right| & \text { if non-degenerate accretor } \\ \min \left(f_{\mathrm{MT}}\left|\dot{m}_{d}\right|, \dot{m}_{\mathrm{Edd}}\right) & \text { otherwise, }\end{cases}
$$

where $\dot{m}_{a}$ is the accretion rate, $\dot{m}_{d}$ is the mass loss rate by the donor, $\dot{m}_{\text {Edd }}$ is the Eddington accretion rate and $f_{\mathrm{MT}} \in(0,1]$ is the accretion efficiency. Here, we consider $f_{\mathrm{MT}}=0.1,0.5,1.0$. The original prescriptions by [128] are close to $f_{\mathrm{MT}}=1.0$. We parametrize common envelope evolution with the parameter $\alpha$ [128]. Here, we consider $\alpha=1,5,10$, large values of $\alpha$ meaning that the envelope is easily ejected, without much shrinking of the binary. In its original meaning [141], $\alpha$ is the fraction of orbital energy that is transferred to the envelope during the spiral-in phase. Here, we also consider values of $\alpha>1$ because the original formalism does not include additional contributions to the energy budget (e.g., [142,143]).

Giacobbo et al. (2018, [30]) have shown (e.g., their Figure 4) that with these prescriptions for stellar and binary evolution the maximum mass of a single $\mathrm{BH}$ can be as high as $m_{\mathrm{BH}} \approx 65-70 \mathrm{M}_{\odot}$. Such massive BHs come from metal-poor stars $(Z \sim 0.0002)$ with initial mass $m_{\mathrm{ZAMS}} \approx 70-80 \mathrm{M}_{\odot}$, which retain most of their hydrogen envelope at the time of 
collapse and have sufficiently small helium cores to avoid pulsational pair instability [107]. A metallicity $Z \sim 0.0002 \approx 0.01 \mathrm{Z} \odot$ is typical of population II stars (the most metal poor GCs in the Milky Way have metallicity $Z \sim 0.0002$ [144]. One of the most metal-poor dwarf galaxies in the local Universe, $I Z w 18$, has metallicity $Z \approx 0.0004$ [145]).

However, the maximum mass of a $\mathrm{BH}$ merging within a Hubble time as a result of isolated binary evolution is only $m_{\mathrm{BH}} \approx 50 \mathrm{M}_{\odot}$ [30]. This happens because binary stars that are sufficiently tight to merge within a Hubble time by GW emission evolve through mass transfer and common envelope. These processes remove the hydrogen envelope, leading to smaller $\mathrm{BH}$ masses. Hence, the resulting BBH cannot have a total mass higher than $m_{\mathrm{TOT}} \approx 100 \mathrm{M}_{\odot}$.

In dynamical environments, exchanges and dynamical hardening might allow even more massive BHs to merge, up to total binary masses $m_{\mathrm{TOT}} \approx 130-140 \mathrm{M}_{\odot}$ [56]. For this reason, we consider two different sets of models for $1 \mathrm{~g}$ masses. In our fiducial model A5F05 (conservative approach), the masses of $1 \mathrm{~g}$ BBHs are randomly drawn from catalogs of BBHs simulated with MOBSE. In this case, the evolution of the semi-major axis $\mathcal{A}$ and of the eccentricity $e$ of the BBHs are calculated as [146]

$$
\begin{gathered}
\frac{\mathrm{d} \mathcal{A}}{\mathrm{d} t}=-\frac{64}{5} \frac{G^{3} m_{1} m_{2}\left(m_{1}+m_{2}\right)}{c^{5} \mathcal{A}^{3}\left(1-e^{2}\right)^{7 / 2}}\left(1+\frac{73}{24} e^{2}+\frac{37}{96} e^{4}\right) \\
\frac{\mathrm{d} e}{\mathrm{~d} t}=-\frac{304}{15} e \frac{G^{3} m_{1} m_{2}\left(m_{1}+m_{2}\right)}{c^{5} \mathcal{A}^{4}\left(1-e^{2}\right)^{5 / 2}}\left(1+\frac{121}{304} e^{2}\right),
\end{gathered}
$$

where $G$ is the gravity constant, $c$ is the speed of light, $m_{1}$ and $m_{2}$ are the masses of the primary and secondary $\mathrm{BH}$, respectively.

In the HIGH_MASS model (optimistic approach), the masses of field BBHs are still taken from catalogs of BBH mergers, while the masses of $1 \mathrm{~g}$ dynamical BBHs are uniformly drawn from the list of all the BHs formed with MOBSE, which include both single and binary $\mathrm{BHs}$, both merging and non-merging systems. This ensures that the masses of dynamically formed $1 \mathrm{~g}$ BBHs can reach $m_{\mathrm{TOT}} \approx 140 \mathrm{M}_{\odot}$, while the maximum total mass of field binaries is $m_{\mathrm{TOT}} \approx 100 \mathrm{M}_{\odot}$. In the HIGH_MASS case, we randomly pair the primary and the secondary component and we randomly draw the delay time (i.e., the time elapsed from the formation of the BBH to its merger) from a distribution $d N / d t \propto t^{-1}$ between $t_{\min }=10^{7}$ yr and $t_{\max }=1.4 \times 10^{10} \mathrm{yr}[22,56]$.

We define the dimensionless spin magnitude $a$ as $a \equiv \mathcal{S} c /\left(G m_{\mathrm{BH}}^{2}\right)$, where $\mathcal{S}$ is the spin magnitude in physical units. Spin magnitudes of $1 \mathrm{~g}$ BHs are randomly drawn from a Maxwellian distribution with fiducial one-dimension root-mean square $\sigma_{a}=0.2$ and truncated at $a=1$. We consider also two extreme cases in which $\sigma_{a}=0.01$ (LOW_SPIN model) and $\sigma_{a}=0.4$ (HIGH_SPIN model). This is just a toy model because the uncertainties on BH spin magnitudes from stellar evolution and core-collapse supernova models are still too large to make predictive statements. Angular momentum transport via the magnetic Tayler-Spruit instability might be effective and lead to predominantly low spins (e.g., $[147,148])$, while binary evolution processes can significantly affect the overall picture $[149,150]$. Our LOW_SPIN case can be interpreted as the result of the spin distribution inferred by [147]. Spin directions in dynamical BBHs are isotropically distributed over a sphere [62].

Our set of runs is described in Table 1 . The initial MOBSE population of each model is obtained running $1.2 \times 10^{8}$ binary stars with metallicity $Z=0.02,0.016,0.012,0.008$, $0.006,0.004,0.002,0.0016,0.0012,0.0008,0.0004,0.0002$. The initial mass of the primary is drawn from a Kroupa initial mass function [151] between 5 and $150 \mathrm{M}_{\odot}$. Mass ratios, orbital periods and eccentricities are randomly drawn following the distributions presented in [152]. 
Table 1. Main properties of the runs presented in this paper.

\begin{tabular}{|c|c|c|c|c|c|c|c|}
\hline Run Name & $\begin{array}{c}\alpha \\
1 \mathrm{~g}\end{array}$ & $\begin{array}{c}f_{\mathrm{MT}} \\
1 \mathrm{~g}\end{array}$ & $\begin{array}{l}t_{\mathrm{del}} \\
1 \mathrm{~g}\end{array}$ & $\begin{array}{l}\sigma_{a} \\
1 \mathrm{~g}\end{array}$ & $\begin{array}{l}m_{2} \\
N g\end{array}$ & $\begin{array}{c}t_{\min }[\mathrm{Myr}] \\
\mathrm{Ng}\end{array}$ & $\begin{array}{c}\log _{10}\left(v_{\mathrm{esc}} / \mathrm{km} \mathrm{s}^{-1}\right) \\
\text { NSC, GC, YSC }\end{array}$ \\
\hline Fiducial, A5F05 & 5.0 & 0.5 & Equation (6) & 0.2 & uniform & 10 & $2 \pm 0.2,1.3 \pm 0.2,0.7 \pm 0.2$ \\
\hline A5F01 & 5.0 & 0.1 & Equation (6) & 0.2 & uniform & 10 & $2 \pm 0.2,1.3 \pm 0.2,0.7 \pm 0.2$ \\
\hline A5F1 & 5.0 & 1.0 & Equation (6) & 0.2 & uniform & 10 & $2 \pm 0.2,1.3 \pm 0.2,0.7 \pm 0.2$ \\
\hline A1F01 & 1.0 & 0.1 & Equation (6) & 0.2 & uniform & 10 & $2 \pm 0.2,1.3 \pm 0.2,0.7 \pm 0.2$ \\
\hline A1F05 & 1.0 & 0.5 & Equation & 0.2 & uniform & 10 & $2 \pm 0.2,1.3 \pm 0.2,0.7 \pm 0.2$ \\
\hline A1F1 & 1.0 & 1.0 & Equation (6) & 0.2 & uniform & 10 & $2 \pm 0.2,1.3 \pm 0.2,0.7 \pm 0.2$ \\
\hline A10F01 & 10.0 & 0.1 & Equation (6) & 0.2 & uniform & 10 & $2 \pm 0.2,1.3 \pm 0.2,0.7 \pm 0.2$ \\
\hline A10F05 & 10.0 & 0.5 & Equation (6) & 0.2 & uniform & 10 & $2 \pm 0.2,1.3 \pm 0.2,0.7 \pm 0.2$ \\
\hline A10F1 & 10.0 & 1.0 & Equation (6) & 0.2 & uniform & 10 & $2 \pm 0.2,1.3 \pm 0.2,0.7 \pm 0.2$ \\
\hline HIGH_MASS & - & - & $t^{-1}$ & 0.2 & uniform & 10 & $2 \pm 0.2,1.3 \pm 0.2,0.7 \pm 0.2$ \\
\hline SMALL_M2 & 5.0 & 0.5 & Equation (6) & 0.2 & MOBSE & 10 & $2 \pm 0.2,1.3 \pm 0.2,0.7 \pm 0.2$ \\
\hline LOW_SPIN & 5.0 & 0.5 & Equation (6) & 0.01 & uniform & 10 & $2 \pm 0.2,1.3 \pm 0.2,0.7 \pm 0.2$ \\
\hline HIGH_SPIN & 5.0 & 0.5 & Equation (6) & 0.4 & uniform & 10 & $2 \pm 0.2,1.3 \pm 0.2,0.7 \pm 0.2$ \\
\hline SHORT_DELA & 5.0 & 0.5 & Equation (6) & 0.2 & uniform & 0.1 & $2 \pm 0.2,1.3 \pm 0.2,0.7 \pm 0.2$ \\
\hline LONG_DELAY & 5.0 & 0.5 & Equation (6) & 0.2 & uniform & 100 & $2 \pm 0.2,1.3 \pm 0.2,0.7 \pm 0.2$ \\
\hline BROAD_VESC & 5.0 & 0.5 & Equation (6) & 0.2 & uniform & 10 & $2 \pm 0.3,1.3 \pm 0.3,0.7 \pm 0.3$ \\
\hline NARROW̄_VESC & 5.0 & 0.5 & Equation (6) & 0.2 & uniform & 10 & $2 \pm 0.1,1.3 \pm 0.1,0.7 \pm 0.1$ \\
\hline
\end{tabular}

Column 1: Name of the model. Column 2: Parameter $\alpha$ of common envelope for 1g BBHs. Column 3: Parameter $f_{\mathrm{MT}}$ of accretion efficiency for non-degenerate accretors (Equation (5)) in the case of $1 \mathrm{~g}$ BBHs. Column 4: Delay time distribution of $1 \mathrm{~g} \mathrm{BBHs;} \mathrm{'Equation} \mathrm{(6)'} \mathrm{indicates} \mathrm{that} \mathrm{the} \mathrm{delay} \mathrm{times} \mathrm{were} \mathrm{calculated} \mathrm{solving} \mathrm{Equation} \mathrm{(6);}$ ' $t^{-1}$ ' means that delay times were randomly drawn from $d N / d t \propto t^{-1}$. Column 5: One-dimensional root-mean square associated with the Maxwellian distribution used to extract $1 \mathrm{~g}$ spin magnitudes; we adopted values $\sigma_{a}=0.2$ (fiducial), 0.01 (LOW_SPIN), 0.4 (HIGH_SPIN). Column 6: Distribution from which we drew the mass of the secondary component in the $\mathrm{Ng} \mathrm{BBHs;} \mathrm{'uniform'} \mathrm{means} \mathrm{that} m_{2}$ is uniformly distributed between $m_{\mathrm{MIN}}=3 \mathrm{M}_{\odot}$ and $m_{\mathrm{MAX}}=m_{1}$ (fiducial), 'MOBSE' means that we randomly selected $m_{2}$ from catalogs of $\mathrm{BHs}$ simulated with MOBSE (used in the SMALL_M2 run). Column 7: $t_{\min }$ is the minimum delay time for Ng BBHs. Column 8: Mean and standard deviation of the lognormal distribution of escape velocities $v_{\text {esc }}$ for NSCs, GCs and YSCs.

\subsection{Relativistic Kicks}

We model the magnitude of relativistic kicks according to Equation (12) of [98]:

$$
v_{\text {kick }}=\left(v_{m}^{2}+v_{\perp}^{2}+2 v_{m} v_{\perp} \cos \xi+v_{\|}^{2}\right)^{1 / 2}
$$

where

$$
\begin{array}{r}
v_{m}=A \eta^{2} \frac{(1-q)}{(1+q)}(1+B \eta) \\
v_{\perp}=H \frac{\eta^{2}}{(1+q)}\left|a_{1 \|}-q a_{2 \|}\right| \\
v_{\|}=\frac{16 \eta^{2}}{(1+q)}\left[V_{1,1}+V_{A} S_{\|}+V_{B} S_{\|}^{2}+V_{C} S_{\|}^{3}\right] \\
\left|a_{1 \perp}-q a_{2 \perp}\right| \cos \left(\phi_{\Delta}-\phi\right) .
\end{array}
$$

In the above equations, $q=m_{2} / m_{1}$ with $m_{2} \leq m_{1}, \eta=q(1+q)^{-2}, A=1.2 \times$ $10^{4} \mathrm{~km} \mathrm{~s}^{-1}, B=-0.93, H=6.9 \times 10^{3} \mathrm{~km} \mathrm{~s}^{-1},\left(V_{1,1}, V_{A}, V_{B}, V_{C}\right)=(3678,2481,1792$, 1506) $\mathrm{km} \mathrm{s}^{-1}, \xi=145^{\circ}$ [153], while $\vec{a}_{1}$ and $\vec{a}_{2}$ are the spin vectors of the primary and secondary BHs, respectively. Moreover, $a_{1 \|}\left(a_{2 \|}\right)$ is the component of the spin of the primary (secondary) BH parallel to the orbital angular momentum of the binary system, while $a_{1 \perp}\left(a_{2 \perp}\right)$ is the component of the spin of the primary (secondary) BH lying in the orbital plane. $S_{\|}$is the component parallel to the orbital angular momentum of the vector $\vec{S}=2\left(\vec{a}_{1}+q^{2} \vec{a}_{2}\right) /(1+q)^{2}$. Finally, $\phi_{\Delta}$ represents the angle between the direction of the infall at merger (which we randomly draw in the BBH orbital plane) and the in-plane component of $\vec{\Delta} \equiv\left(m_{1}+m_{2}\right)^{2}\left(\vec{a}_{1} q \vec{a}_{2}\right) /(1+q)$, while $\phi$ is the phase of the BBH, randomly drawn between 0 and $2 \pi$. Equation (7) results from an empirical model for the recoil velocity as a function of the progenitor's parameters (mostly $q, \vec{a}_{1}$ and $\vec{a}_{2}$ ). The basic idea behind it is that the recoil of spinning BHs is mostly produced close to the time of 
merger [154], and can be modeled by a parametrized dependence of the leading (on spins and mass ratio) post-Newtonian expressions for the linear momentum radiated [155]. The term $v_{\mathrm{m}}$ mainly comes from the contribution of the mass ratio $q$ to the linear momentum radiated (the merger of an unequal mass $\mathrm{BBH}$ produces a kick even if the two BHs are non spinning), while $v_{\perp}$ and $v_{\|}$account for the contribution of the spin components aligned and orthogonal to the orbital angular momentum, respectively. The coefficients in Equation (7) are given by fits to full numerical relativity simulations, as detailed in [98]. This formalism yields kicks up to $\sim 4000 \mathrm{~km} \mathrm{~s}^{-1}$, but the most common kicks are of the order of a few hundred $\mathrm{km} \mathrm{s}^{-1}$, as shown in Figure 9 of [98] and discussed in Section 3.1.

\subsection{Escape Velocities}

For each merger, we calculate the relativistic kick magnitude as in Equation (7) and then compare it with the escape velocity of the host cluster $v_{\text {esc }}$. If $v_{\text {kick }}<v_{\text {esc }}$, the remnant is retained inside its host cluster and can undergo another merger. Otherwise, it is ejected and remains a single $\mathrm{BH}$. We randomly draw $v_{\text {esc }}$ from a log-normal distribution with median $\left\langle\log _{10}\left(v_{\text {esc }} / \mathrm{km} \mathrm{s}^{-1}\right)\right\rangle=2.0,1.3,0.7$ (standard deviation $\sigma_{\mathrm{v}}=0.2,0.2,0.2$ ) for NSCs, GCs, and YSCs, respectively. This choice is motivated by observations of NSCs [71], GCs [156] and YSCs [127] in the local Universe. Figure 1 shows the distribution of escape velocities in our fiducial case. In the next sections, we show what happens if we change these assumptions. Namely, in the BROAD_VESC (NARROW_VESC) model we assume $\sigma_{\mathrm{v}}=0.3(0.1)$ for NSCs, GCs and YSCs.

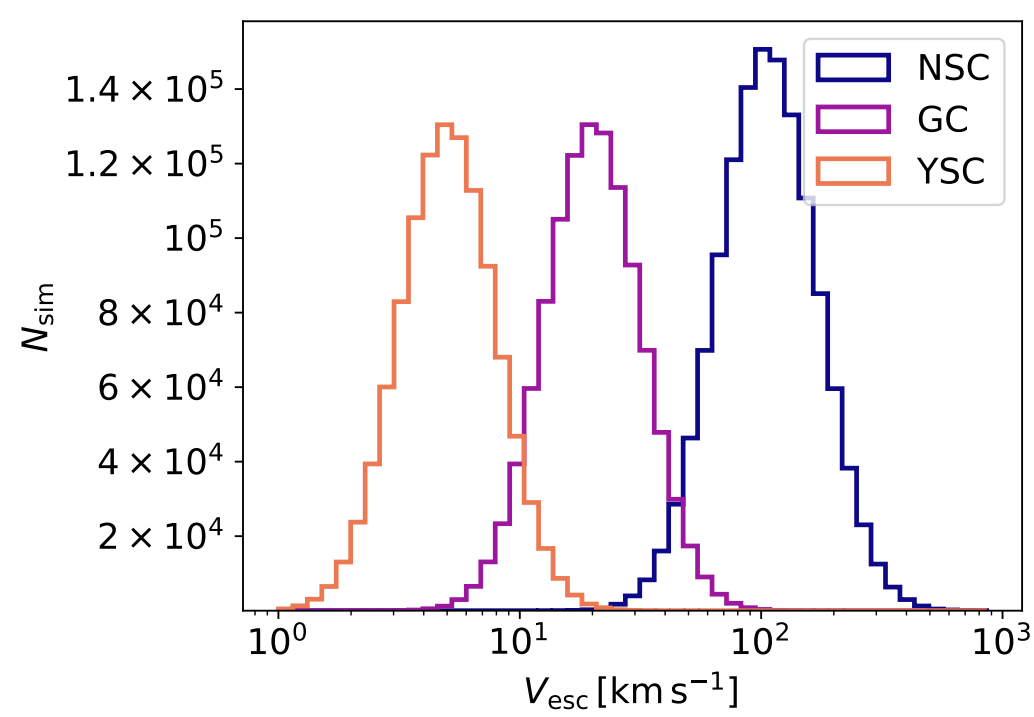

Figure 1. Distribution of escape velocities adopted in the fiducial case. See Section 2.3.

\subsection{Nth Generation ( $\mathrm{Ng}$ ) Mass and Spin}

We model the mass and spin of merger remnants using the fitting formulas in [157] for quasi-circular non-precessing mergers (see also $[76,158,159])$. The final mass is $\approx 0.95$ the total mass of the two merging BHs, while the final spin magnitude clusters around $a_{\mathrm{f}} \approx 0.75$. If the merger remnant is retained, it eventually pairs up with another $\mathrm{BH}$. The mass of the companion is selected in two different ways. To account for the fact that the secondary component might be either a $1 \mathrm{~g}$ or an $\mathrm{Ng}$ object with $N>1$, we uniformly draw the mass of the secondary $\mathrm{BH}, m_{2}$, between $m_{\mathrm{MIN}}=3 \mathrm{M}_{\odot}$ and $m_{\mathrm{MAX}}=m_{1}$ (fiducial model). This assumption favors $\mathrm{Ng}-\mathrm{Ng}$ mergers with respect to $\mathrm{Ng}-1 \mathrm{~g}$ mergers. To account for cases in which the primary component is an $N g$ merger (with $N>1$ ) and the secondary component is a $1 \mathrm{~g} \mathrm{BH}$, we draw the mass of the secondary $\mathrm{BH}$ from the population-synthesis catalogs of $1 \mathrm{~g}$ BHs (model SMALL_M2).

The spins of secondary BHs are randomly drawn from a Maxwellian distribution with default one-dimensional root-mean square $\sigma_{a}=0.2$. In the LOW_SPIN (HIGH_SPIN) 
case, $\sigma_{a}=0.01(0.4)$. This is a simplification because we do not distinguish whether the secondary component is an $\mathrm{Ng}$ or a $1 \mathrm{~g} \mathrm{BH}$. The spin vectors of both the primary and secondary $\mathrm{BH}$ are isotropically distributed over a sphere.

Finally, in all models we check that the mass of the remnant $\mathrm{BH}$ is always less than

$$
m_{\mathrm{th}}=10^{-3} m_{\mathrm{SC}} \approx 10^{3} \mathrm{M}_{\odot}\left(\frac{v_{\mathrm{esc}}}{100 \mathrm{~km} \mathrm{~s}^{-1}}\right)^{2} .
$$

This condition is equivalent to assuming that the most massive $\mathrm{BH}$ cannot be more massive than the total mass of all BHs in the star cluster, assuming a Kroupa IMF. If a BH hits this mass threshold, it cannot grow any further by hierarchical merger.

\subsection{Delay Times of $\mathrm{Ng}$ Mergers}

For $\mathrm{Ng}$ mergers (where $N=2$ or more), we randomly draw the delay times according to a distribution uniform in $d N / d t \propto t^{-1}$ [22] and spanning from $t_{\min }=[0.1,10,100]$ Myr (see column 7 of Table 1 ) to $t_{\max }=1.4 \times 10^{4}$ Myr. By adopting $d N / d t \propto t^{-1}$, we have assumed that GW decay is the dominant effect to determine the delay time. Since the GW timescale $\tau_{\mathrm{GW}} \propto \mathcal{A}^{4}$, where $\mathcal{A}$ is the initial semi-major axis of the $\mathrm{BBH}$ [146], for an initial semi-major axis distribution $f(\mathcal{A}) \propto \mathcal{A}^{-1}$, we obtain a delay time distribution $d N / d t \propto t^{-1}$ [160-162]. This is the crudest assumption in our method because we neglect the impact of dynamical hardening on the evolution of the BBH semi-major axis. However, this assumption is supported by $N$-body simulations of dense YSCs $[55,56]$, which show that dynamical BBH mergers follow a trend $d N / d t \propto t^{-1}$. The choice of $t_{\text {min }}$ depends on the time for dynamical pairing of the $\mathrm{BBH} t_{\mathrm{dyn}}$, i.e., the time needed for a single $\mathrm{BH}$ to find a new companion $\mathrm{BH}$ via dynamical interactions. This can be estimated as the sum of the dynamical friction timescale $\left(t_{\mathrm{DF}}\right.$, i.e., the time over which the merger remnant, which is ejected in the outskirts of the star cluster by the relativistic kick, sinks back to the core of the parent cluster by dynamical friction, [163]) and the three-body timescale $\left(t_{3 b b}\right.$, i.e., the timescale for BBH formation by three-body encounters, [164]):

$$
\begin{aligned}
t_{\mathrm{DF}} & =9.5 \mathrm{Myr}\left(\frac{m_{\mathrm{BH}}}{30 M_{\odot}}\right)^{-1}\left(\frac{M_{\mathrm{SC}}}{10^{6} M_{\odot}}\right)\left(\frac{n}{10^{6} \mathrm{pc}^{-3}}\right)^{-1 / 2}, \\
t_{3 \mathrm{bb}} & =0.1 \mathrm{Myr}\left(\frac{n}{10^{6} \mathrm{pc}^{-3}}\right)^{-2}\left(\frac{\sigma_{\mathrm{SC}}}{30 \mathrm{~km} \mathrm{~s}^{-1}}\right)^{9}\left(\frac{m_{\mathrm{BH}}}{30 M_{\odot}}\right)^{-5},
\end{aligned}
$$

where $M_{\mathrm{SC}}$ and $n$ are the star cluster mass and central number density, while $\sigma_{\mathrm{SC}}=v_{\mathrm{esc}} /(2 \sqrt{3})$ is the one-dimensional velocity dispersion. In Equation (10), we assumed that the average mass of a star in the star cluster is $1 \mathrm{M}_{\odot}$ and that, locally, the star cluster is in equipartition [165]. The timescale for dynamical BBH formation is then $t_{\mathrm{dyn}}=t_{\mathrm{DF}}+t_{3 \mathrm{bb}}$. For most star clusters, we find $0.1 \leq t_{\mathrm{dyn}} / \mathrm{Myr} \leq 100$, roughly corresponding to the values of $t_{\min }$ we assume in our analysis (column 7 of Table 1 ).

\subsection{Summary of the Models}

In Table 1, A5F05 is our fiducial model. Models with name $\mathrm{A} i \mathrm{~F} j$ (with $i=1,5,10$ and $j=01,05,1)$ differ from the fiducial model only for the choice of the common envelope parameter $\alpha(\alpha=1,5,10$ if $i=1,5,10)$ and of the accretion efficiency $f_{\mathrm{MT}}\left(f_{\mathrm{MT}}=0.1,0.5\right.$, 1 if $j=01,05,1)$. The model HIGH_MASS differs from the fiducial model for the choice of the masses of $1 \mathrm{~g}$ BHs and for their delay time distribution. In the model HIGH_MASS, $1 \mathrm{~g} \mathrm{BH}$ masses in star clusters are uniformly sampled from all BHs generated with MOBSE (including single BHs), mimicking the impact of dynamical exchanges. Delay times are drawn from $d N / d t \propto t^{-1}$.

The SMALL_M2 model differs from the fiducial one for the masses $m_{2}$ of secondary $\mathrm{BH}$ in $\mathrm{Ng}$ mergers, which are randomly drawn from $1 \mathrm{~g} \mathrm{BHs}$. Hence, in this model all $\mathrm{BBH}$ mergers occur with a $1 \mathrm{~g}$ secondary $\mathrm{BH}$. In contrast, $m_{2}$ is uniformly sampled in $\left[3 \mathrm{M}_{\odot}, m_{1}\right]$ in all the other models. 
The LOW_SPIN and HIGH_SPIN models differ from the fiducial model only for the distribution of spin magnitudes of $1 \mathrm{~g}$ BHs. The one-dimensional root-mean square $\sigma_{a}$ is 0.01 and 0.4 in LOW_SPIN and HIGH_SPIN, respectively.

The SHORT_DELAY and LONG_DELAY models differ only for the minimum value of the delay time $t_{\min }$ of $\mathrm{Ng}$ mergers, which is 0.1 and $100 \mathrm{Myr}$, respectively. Finally, the BROAD_VESC and NARROW_VESC models differ from the fiducial case for the standard deviation of the log-normal distribution of $v_{\mathrm{esc}}$, which is 0.3 and 0.1 in the former and in the latter case.

\subsection{Merger Rate}

We calculate the merger rate by assuming that each channel accounts for a fraction $f_{i}(t)$ of the star formation rate density at a given look-back time $t$ (where $i=$ NSC, GC, YSC or field).

In our fiducial model, we assume that $f_{\mathrm{GC}}(t)$ is given by

$$
f_{\mathrm{GC}}(t)=f_{\max , \mathrm{GC}} \exp \left[-\frac{\left(t-t_{\mathrm{GC}}\right)^{2}}{2 \sigma_{t}^{2}}\right],
$$

where $f_{\max , \mathrm{GC}}=0.1, t_{\mathrm{GC}}=11.8 \mathrm{Gyr}$ and $\sigma_{t}=2.5 \mathrm{Gyr}$. The parameters $t_{\mathrm{GC}}$ and $\sigma_{t}$ are chosen based on the age distribution of Galactic GCs [166-168].

NSCs likely are the result of the dynamical assembly of GCs, which sink to the center of the galactic potential well by dynamical friction [169-175], plus some contribution from in situ star formation [176]. Hence, we assume the same functional form for $f_{\mathrm{NSC}}(t)$, with a different normalization:

$$
f_{\mathrm{NSC}}(t)=f_{\mathrm{max}, \mathrm{NSC}} \exp \left[-\frac{\left(t-t_{\mathrm{GC}}\right)^{2}}{2 \sigma_{t}^{2}}\right],
$$

where $f_{\max , \mathrm{NSC}}=0.01$, while $t_{\mathrm{GC}}$ and $\sigma_{t}$ are the same as in Equation (11). The values of both $f_{\max , \mathrm{GC}}$ and $f_{\mathrm{max}, \mathrm{NSC}}$ are calibrated to give a mass budget of GCs and NSCs that matches the observed ones at low redshifts $[156,177]$.

To keep the fiducial model as simple as possible, we assume

$$
f_{\mathrm{YSC}}(t)=\min \left\{0.3,\left[1-f_{\mathrm{GC}}(t)-f_{\mathrm{NSC}}(t)\right]\right\} .
$$

Finally, we define $f_{\text {field }}(t)=\max \left\{0,\left[1-f_{\mathrm{GC}}(t)-f_{\mathrm{NSC}}(t)-f_{\mathrm{YSC}}(t)\right]\right\}$. In the following sections, we briefly discuss the impact of changing the $f_{i}(t)$ parameters on the merger rate.

The total merger rate for each channel is then evaluated as

$$
\mathcal{R}_{i}(z)=\frac{\mathrm{d}}{\mathrm{d} t(z)} \int_{z_{\max }}^{z} f_{i}\left(z^{\prime}\right) \psi\left(z^{\prime}\right) \frac{\mathrm{d} t\left(z^{\prime}\right)}{\mathrm{d} z^{\prime}} \mathrm{d} z^{\prime} \int_{Z_{\min }\left(z^{\prime}\right)}^{Z_{\max }\left(z^{\prime}\right)} \eta(Z) \mathcal{F}\left(z^{\prime}, z, Z\right) \mathrm{d} Z,
$$

where $t(z)$ is the look-back time at redshift $z, \psi\left(z^{\prime}\right)$ is the cosmic star formation rate density at redshift $z^{\prime}, f_{i}\left(z^{\prime}\right)$ is the fraction of the total star formation rate that goes into channel $i=$ NSCs, GCs, YSCs or field at redshift $z^{\prime}, Z_{\min }\left(z^{\prime}\right)$ and $Z_{\max }\left(z^{\prime}\right)$ are the minimum and maximum metallicity of stars formed at redshift $z^{\prime}, \eta(Z)$ is the merger efficiency at metallicity $Z$, and $\mathcal{F}\left(z^{\prime}, z, Z\right)$ is the fraction of BBHs that form at redshift $z^{\prime}$ from stars with metallicity $Z$ and merge at redshift $z$, normalized to all BBHs that form from stars with metallicity $Z$. To calculate the look-back time we take the cosmological parameters $\left(H_{0}\right.$, $\Omega_{\mathrm{M}}$ and $\Omega_{\Lambda}$ ) from [178]. The maximum considered redshift in Equation (14) is $z_{\max }=15$, which we assume to be the epoch of formation of the first stars. The merger efficiency $\eta(Z)$ is estimated as the number of BBHs that merge within a Hubble time in a coeval population 
of star with initial mass $M_{*}$ and metallicity $Z$, divided by $M_{*}$. We take the fitting formula for the star formation rate density $\psi\left(z^{\prime}\right)$ from [179]:

$$
\psi(z)=0.01 \frac{(1+z)^{2.6}}{1+[(1+z) / 3.2]^{6.2}} \mathrm{M}_{\odot} \mathrm{Mpc}^{-3} \mathrm{yr}^{-1}
$$

and we model the metallicity evolution as described in [180]. Equation (15) is based on data ranging from $z=0$ to $z \sim 10$ [179]. In future work, we will consider alternative options to model the very high redshift star formation, such as modelling population III stars separately [181] and considering high-redshift long gamma-ray bursts as tracers of star formation ([182], but see [183] for some caveats).

\section{Results}

\subsection{Properties of Hierarchical Mergers}

Figure 2 shows the mass of the primary $\mathrm{BH}\left(m_{1}\right)$, the mass of the secondary $\mathrm{BH}\left(m_{2}\right)$, the spin magnitude of the primary $\mathrm{BH}\left(a_{1}\right)$ and the kick velocity $\left(v_{\text {kick }}\right)$ in the fiducial model (A5F05) for $Z=0.0002$. The maximum primary and secondary mass strongly depend on the environment: We have $2 \mathrm{~g}$ BBHs even in YSCs and GCs, but NSCs are more effective in producing hierarchical mergers because of the larger value of $v_{\text {esc }}$. In the fiducial model, the maximum primary mass is $\approx 100 \mathrm{M}_{\odot}$ in YSCs and GCs, while it is close to $\approx 10^{3} \mathrm{M}_{\odot}$ in NSCs. The distribution of primary spin magnitudes shows a clear secondary peak at $a_{1} \approx 0.7-0.8$ in both GCs and NSCs, corresponding to the typical values of $\mathrm{Ng}$ merger remnants. The most common kick velocities are $v_{\text {kick }} \sim 100-300 \mathrm{~km} \mathrm{~s}^{-1}$, but larger kicks, up to $\sim 3000 \mathrm{~km} \mathrm{~s}^{-1}$, are possible.
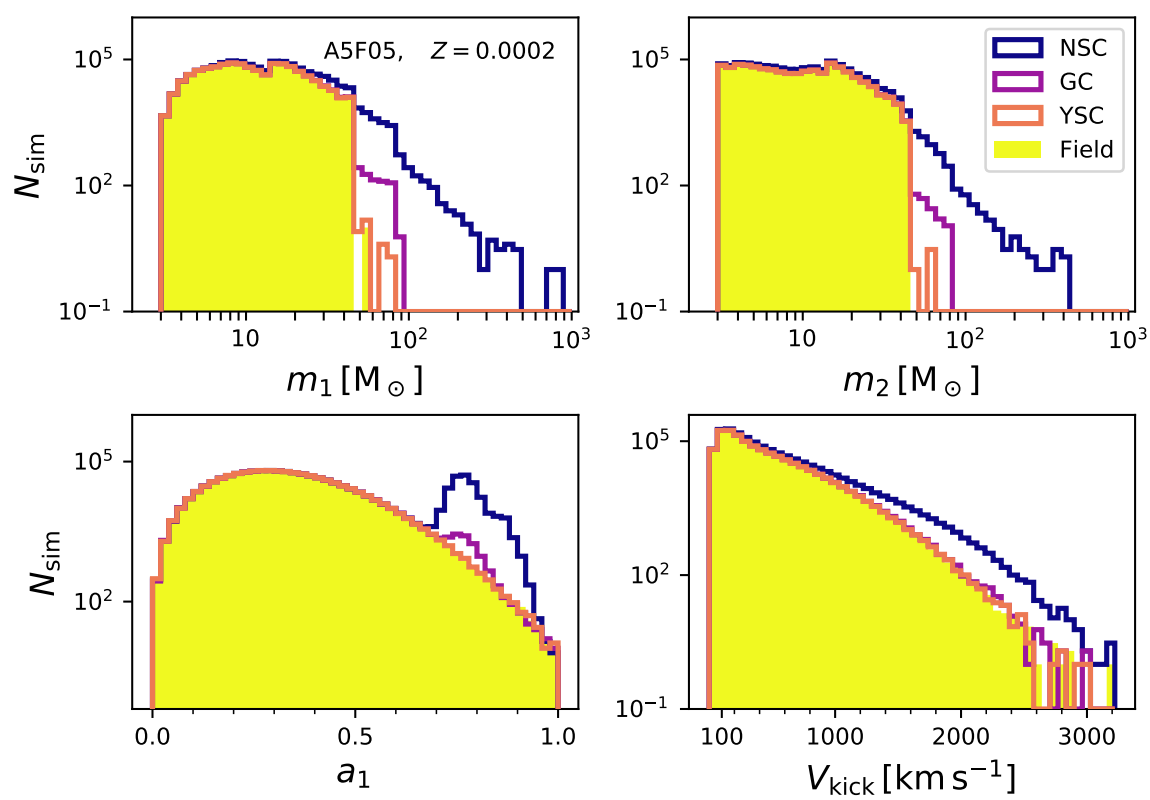

Figure 2. Upper left (right): primary (secondary) mass distribution in the fiducial model A5F05 for $Z=0.0002$. Lower left (right): Primary spin magnitude $a_{1}$ (relativistic kick velocity $v_{\text {kick }}$ ) in the fiducial case for $Z=0.0002$. The distributions for each channel are drawn from an initial (i.e., zero-age main sequence) stellar population of $1.5 \times 10^{10} \mathrm{M}_{\odot}$, assuming a binary fraction $f_{\text {bin }}=0.5$.

Figures 3 and 4 compare the primary mass distributions that we obtain by varying the values of $\alpha$ and $f_{\mathrm{MT}}$ in the first generation of BHs for metallicity $Z=0.0002$ and 0.002, respectively. By comparing Figures 3 and 4, it is apparent that both the BH mass distribution and the maximum BH mass strongly depend on progenitor's metallicity, even in hierarchical mergers. Moreover, the efficiency of common-envelope ejection $\alpha$ and the efficiency of mass accretion $f_{\mathrm{MT}}$ significantly affect the mass distribution of $\mathrm{Ng}$ BHs. Hence, 
the mass distribution of $1 \mathrm{~g}$ BBHs, which strongly depends on metallicity, has a crucial impact on the mass distribution of $\mathrm{Ng}$ BHs.
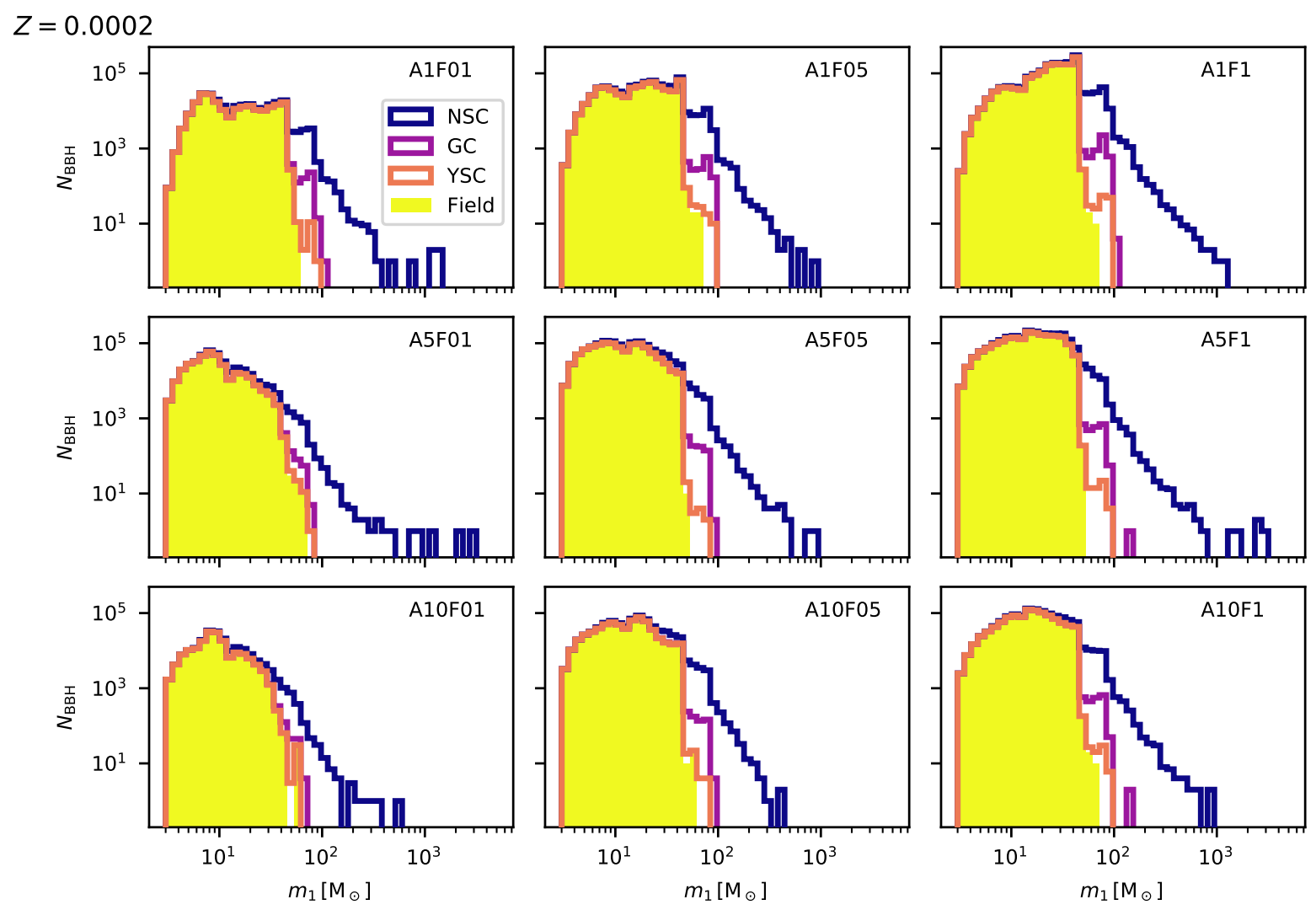

Figure 3. From top to bottom and from left to right: distribution of primary BH masses $\left(m_{1}\right)$, assuming progenitor metallicity $\mathrm{Z}=0.0002$, in the cases A1F01 $\left(\alpha=1, f_{\mathrm{MT}}=0.1\right), \operatorname{A1F05}\left(\alpha=1, f_{\mathrm{MT}}=0.5\right), \operatorname{A1F} 1\left(\alpha=1, f_{\mathrm{MT}}=1\right), \mathrm{A} 5 \mathrm{~F} 01(\alpha=5$, $\left.f_{\mathrm{MT}}=0.1\right), \mathrm{A} 5 \mathrm{~F} 05\left(\alpha=5, f_{\mathrm{MT}}=0.5\right.$, fiducial case $), \mathrm{A} 5 \mathrm{~F} 1\left(\alpha=5, f_{\mathrm{MT}}=1\right), \mathrm{A} 10 \mathrm{~F} 01\left(\alpha=10, f_{\mathrm{MT}}=0.1\right), \mathrm{A} 10 \mathrm{~F} 05(\alpha=10$, $\left.f_{\mathrm{MT}}=0.5\right)$, and $\mathrm{A} 10 \mathrm{~F} 1\left(\alpha=10, f_{\mathrm{MT}}=1\right)$. The distributions for each channel are drawn from an initial (i.e., zero-age main sequence) stellar population of $1.5 \times 10^{10} \mathrm{M}_{\odot}$, assuming a binary fraction $f_{\text {bin }}=0.5$.

In Figure 5, we fix $\alpha=5, f_{\mathrm{MT}}=0.5$ and $Z=0.0002$, and consider the impact of the other main parameters of our model. Drawing the mass of $1 \mathrm{~g}$ BBHs from the distribution of all $1 \mathrm{~g} \mathrm{BHs}$ (including single BHs) shifts the entire distribution of dynamical mergers to higher masses. In the model HIGH_MASS, the most common primary mass of dynamical BBHs is $\sim 30-50 \mathrm{M}_{\odot}$, while the primary masses of field BBHs peak at $\sim 10 \mathrm{M}_{\odot}$. The reason is that MOBSE allows the formation of BHs with mass up to $\sim 65 \mathrm{M}_{\odot}$, but small BHs merge more efficiently than massive $\mathrm{BHs}$ because of the interplay between stellar radii, mass transfer and common envelope evolution. If we randomly pair single BHs from MOBSE data, this effect disappears. Hence, the HIGH_MASS model is realistic if dynamical encounters are very effective, and all BBHs in star clusters form from dynamical exchanges. Based on direct $N$-body simulations coupled with MOBSE, Di Carlo et al. (2020, [56]) have shown that BBHs in YSCs behave in an intermediate way between the HIGH_MASS model and our fiducial model. In the HIGH_MASS model, Ng BHs in both GCs and YSCs can reach masses $m_{1} \sim 200 \mathrm{M}_{\odot}$, while the maximum mass of $\mathrm{Ng}$ BHs in NSCs is $\sim 2000 \mathrm{M}_{\odot}$. 

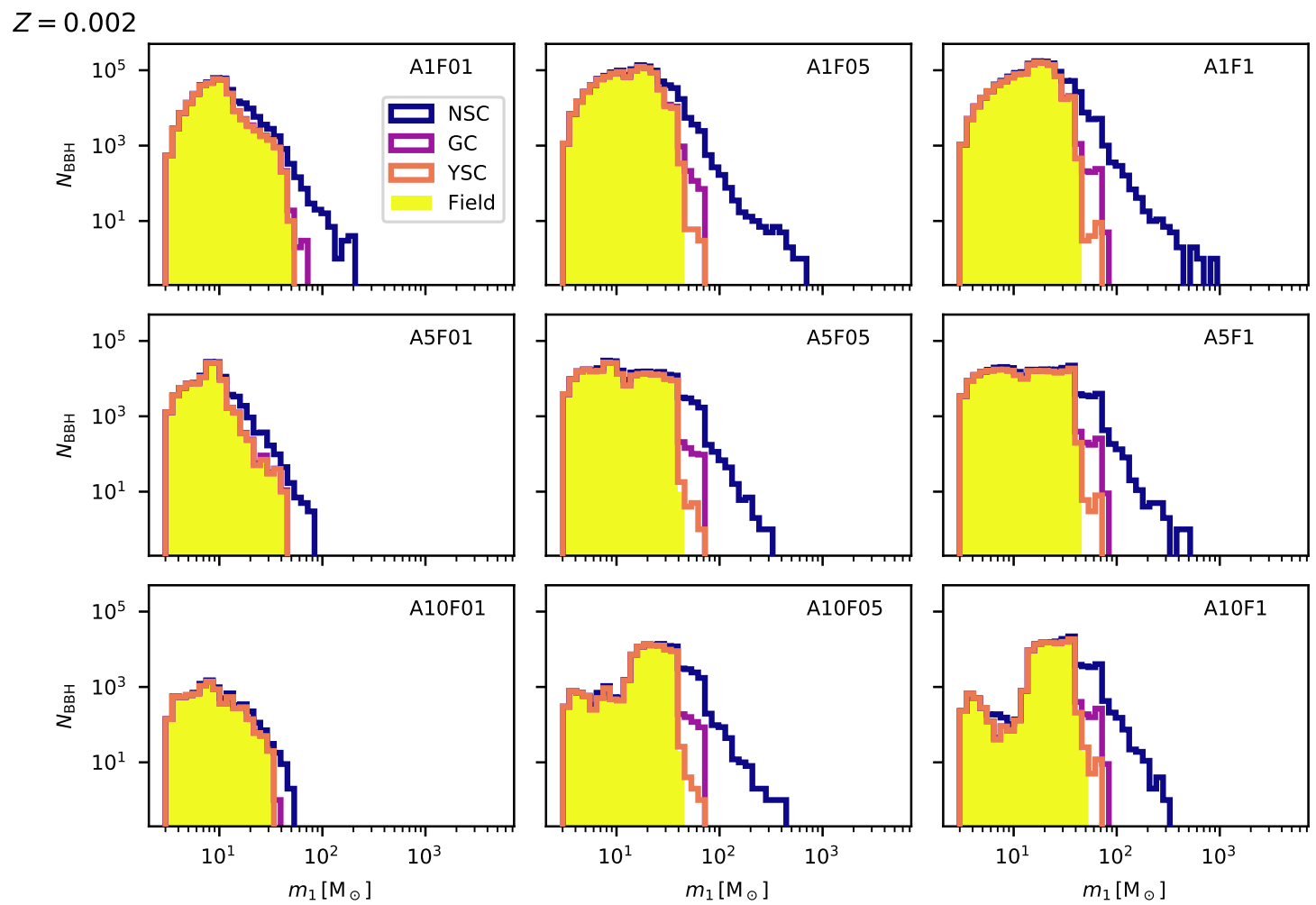

Figure 4. Same as Figure 3 but for progenitor's metallicity $Z=0.002$.
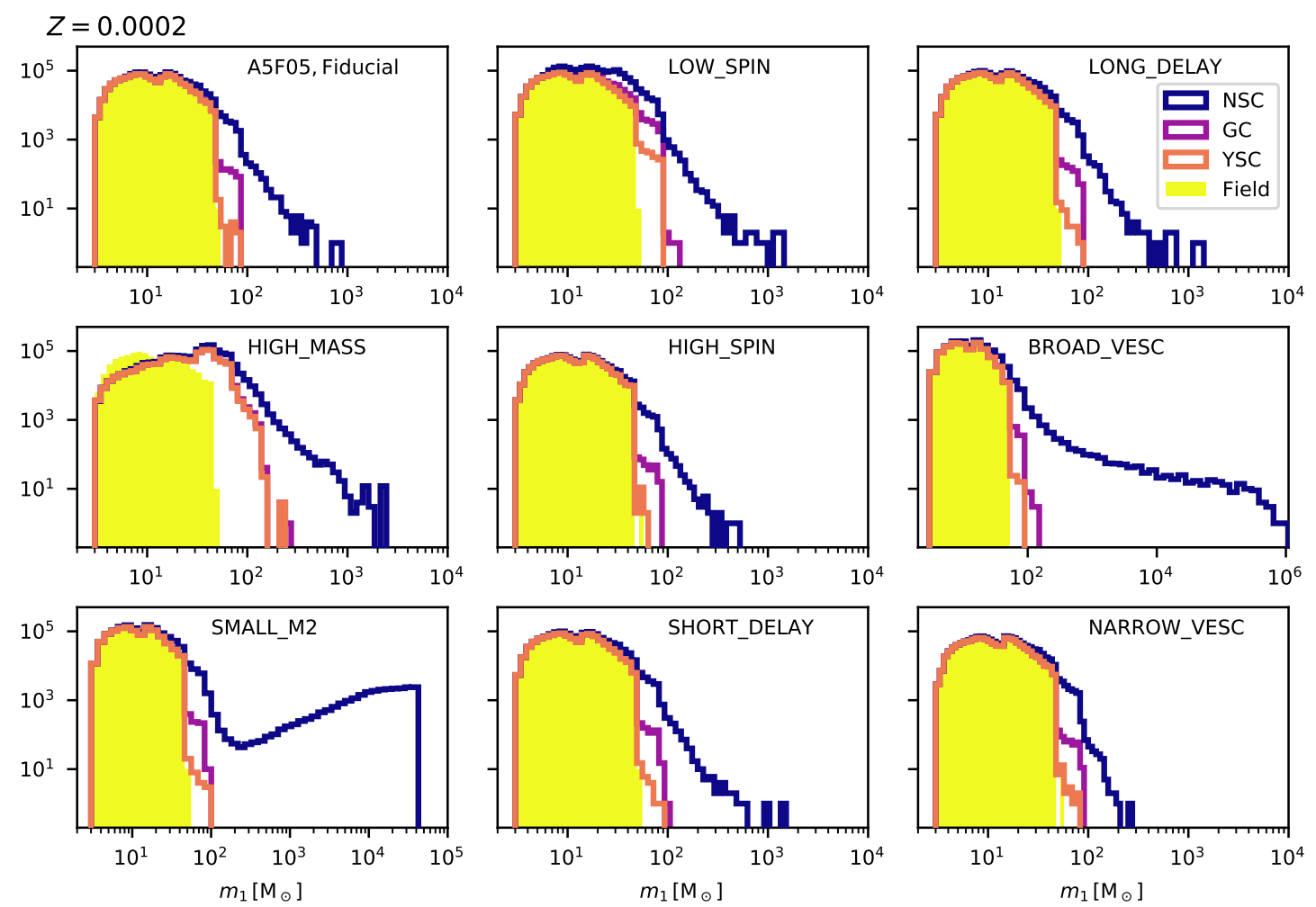

Figure 5. From top to bottom and from left to right: Distribution of primary BH masses $\left(m_{1}\right)$, assuming progenitor metallicity $Z=0.0002$, in the cases A5F05 (fiducial), LOW_SPIN, LONG_DELAY, HIGH_MASS, HIGH_SPIN, BROAD_VESC, SMALL_M2,SHORT_DELAY, and NARROW_VESC. The distributions for each channel are drawn from an initial (i.e., zero-age main sequence) stellar population of $1.5 \times 10^{10} \mathrm{M}_{\odot}$, assuming a binary fraction $f_{\text {bin }}=0.5$. 
In the SMALL_M2 model, we draw the secondary mass from the distribution of $1 \mathrm{~g}$ $\mathrm{BHs}$, which is equivalent to assuming that only $\mathrm{Ng}-1 \mathrm{~g}$ mergers are possible. Hence, this model differs from the others because of the smaller values of $q=m_{2} / m_{1}$ in hierarchical mergers. We observe a peculiar trend of $m_{1}$ in the NSC case: Values of $m_{1} \sim 10^{4} \mathrm{M}_{\odot}$ are about one order of magnitude more common than $m_{1} \sim 200 \mathrm{M}_{\odot}$. The reason is that relativistic kicks get smaller and smaller if $q$ tends to zero. Hence, the maximum mass of the primary $\mathrm{BH}$ in this model is set by the number of hierarchical mergers that happen within a Hubble time, rather than by the relativistic kicks.

If we compare the LOW_SPIN $\left(\sigma_{a}=0.01\right)$ and HIGH_SPIN model $\left(\sigma_{a}=0.4\right)$, we see that high-mass BHs are more and more suppressed if the spin distribution moves to higher values because relativistic kicks get stronger. In contrast, we find only a mild difference between the SHORT_DELAY and LONG_DELAY model, in which we change the minimum delay time $t_{\min }$.

Finally, the escape velocity has a large impact on hierarchical BH masses, especially for NSCs. BHs with mass up to $\sim 10^{6} \mathrm{M}_{\odot}$ form if $\sigma_{\mathrm{v}}=0.3$ (BROAD_VESC), two orders of magnitude more than if $\sigma_{\mathrm{v}}=0.2$ (fiducial case, A5F05) and three orders of magnitude more than if $\sigma_{\mathrm{v}}=0.1$ (NARROW_VESC). This result is consistent with both [47,102], who report that the maximum BH mass approaches $10^{6} \mathrm{M}_{\odot}$ if $v_{\text {esc }} \geq 300 \mathrm{~km} \mathrm{~s}^{-1}$ are considered. This might be a key ingredient to understand the formation of super-massive BHs and the connection between the mass of the central $\mathrm{BH}$ and its parent galaxy mass/central velocity dispersion [177,184-186].

Figure 6 shows the number of BBHs we simulated per each generation $\mathrm{Ng}$ in the case of $Z=0.0002$ and the maximum primary mass in each generation. We only show NSCs because BHs in GCs and YSCs do not exceed the 5th and 3rd generation, respectively. The maximum number of generations in NSCs ranges from a few to a few thousands. In the fiducial case and in most of the other simulations, the maximum number of generations is $N \sim 10$. Only in three cases we obtain a significantly larger number of generations, namely the BROAD_VESC model ( $\approx 40$ generations), the HIGH_MASS model ( $\approx 50$ generations) and the SMALL_M2 model ( $\approx 5000$ generations). The SMALL_M2 case outnumbers all the other models for the number of generations because of the strong dependence of $v_{\text {kick }}$ on $q$. However, even in this extreme case, the number of $N g$ mergers with $N \geq 20$ is $\sim 10^{5}$ times lower than the number of mergers in the first generation.

The upper panel of Figure 7 shows the fraction of $\mathrm{Ng}$ BBH mergers with $N>1$ with respect to all BBH mergers, defined as $f_{>1 g}=\left(N_{2 g}+N_{3 g}+. .+N_{N g}\right) / N_{\mathrm{BBH}}$, where $N_{1 g}, N_{2 g}, N_{3 g}, . ., N_{N g}$ is the number of $1 \mathrm{~g}, 2 \mathrm{~g}, 3 \mathrm{~g}, . ., \mathrm{Ng}$ BBH mergers and $N_{\mathrm{BBH}}$ is the total number of $\mathrm{BBH}$ mergers summing up all possible generations including the first one. In this figure, $f_{>1 g}$ is only shown for NSCs. In the fiducial model and in NSCs, Ng BBH mergers with $N>1$ are about $16 \%$ of all the BBH mergers, with a small dependence on metallicity. For other models, the percentage of $\mathrm{Ng}$ BBHs can be as low as $~ 8 \%$ (HIGH_SPIN case) or as high as $\sim 40-50 \%$ (LOW_SPIN case). For GCs and YSCs these percentages should be lowered by a factor of $\sim 30$ and $\sim 10^{3}$, respectively. Table 2 reports the values of $f_{>1 g}$ in detail.

Table 2. Values of $f_{>1 g}, f_{\mathrm{PISN}}$ and $f_{\mathrm{IMBH}}$ for different runs.

\begin{tabular}{ccccc}
\hline Run Name & Star Cluster & $f_{>1 g}$ & $f_{\text {PISN }}$ & $f_{\text {IMBH }}$ \\
\hline Fiducial, A5F05 & NSC & $0.16,0.15,0.13$ & $0.007,0.009,0$ & $5 \times 10^{-4}, 5 \times 10^{-4}, 0$ \\
& GC & $0.006,0.007,0.005$ & $3 \times 10^{-4}, 5 \times 10^{-4}, 0$ & $0,0,0$ \\
A5F01 & YSC & $10^{-4}, 2 \times 10^{-4}, 0$ & $5 \times 10^{-6}, 2 \times 10^{-5}, 0$ & $0,0,0$ \\
& NSC & $0.14,0.11,0.13$ & $0.003,7 \times 10^{-5}, 0$ & $2 \times 10^{-4}, 0,0$ \\
& GC & $0.005,0.002,0$ & $2 \times 10^{-4}, 0,0$ & $0,0,0$ \\
A5F1 & YSC & $10^{-4}, 5 \times 10^{-5}, 0$ & $10^{-5}, 0,0$ & $0,0,0$ \\
& NSC & $0.14,0.16,0.15$ & $0.014,0.020,0$ & $8 \times 10^{-4}, 8 \times 10^{-4}, 0$ \\
& GC & $0.004,0.008,0.004$ & $7 \times 10^{-4}, 0.0013,0$ & $5 \times 10^{-7}, 0,0$ \\
\hline
\end{tabular}


Table 2. Cont.

\begin{tabular}{|c|c|c|c|c|}
\hline Run Name & Star Cluster & $f_{>1 g}$ & $f_{\text {PISN }}$ & $f_{\mathrm{IMBH}}$ \\
\hline \multirow{3}{*}{ HIGH_MASS } & YSC & $9 \times 10^{-5}, 3 \times 10^{-4}, 0.004$ & $2 \times 10^{-5}, 4 \times 10^{-5}, 0$ & $0,0,0$ \\
\hline & NSC & $0.26,0.25,0.15$ & $0.075,0.055,0$ & $0.016,0.011,0$ \\
\hline & GC & $0.009,0.007,0.005$ & $0.004,0.003,0$ & $6 \times 10^{-4}, 6 \times 10^{-4}, 0$ \\
\hline \multirow{3}{*}{ SMALL_M2 } & YSC & $9 \times 10^{-5}, 8 \times 10^{-5}, 0$ & $4 \times 10^{-5}, 3 \times 10^{-5}, 0$ & $3 \times 10^{-5}, 3 \times 10^{-5}, 0$ \\
\hline & NSC & $0.18,0.19,0.14$ & $0.009,0.014,0$ & $0.019,0.038,0$ \\
\hline & GC & $0.006,0.007,0.003$ & $3 \times 10^{-4}, 4 \times 10^{-4}, 0$ & $0,0,0$ \\
\hline \multirow{3}{*}{ LOW_SPIN } & YSC & $10^{-4}, 2 \times 10^{-4}, 0.0026$ & $10^{-5}, 10^{-5}, 0$ & $0,0,0$ \\
\hline & NSC & $0.45,0.43,0.37$ & $0.021,0.029,0$ & $9 \times 10^{-4}, 0.0011,0$ \\
\hline & GC & $0.11,0.15,0.11$ & $0.007,0.012,0$ & $2 \times 10^{-6}, 4 \times 10^{-6}, 0$ \\
\hline \multirow{4}{*}{ HIGH_SPIN } & YSC & $0.013,0.020,0.013$ & $0.001,8 \times 10^{-4}, 0$ & $0,0,0$ \\
\hline & NSC & $0.08,0.08,0.09$ & $0.004,0.004,0$ & $2 \times 10^{-4}, 3 \times 10^{-4}, 0$ \\
\hline & $\mathrm{GC}$ & $0.002,0.003,0.003$ & $10^{-4}, 2 \times 10^{-4}, 0$ & $0,0,0$ \\
\hline & YSC & $4 \times 10^{-5}, 4 \times 10^{-5}, 0$ & $9 \times 10^{-7}, 0,0$ & $0,0,0$ \\
\hline \multirow[t]{3}{*}{ SHORT_DELAY } & NSC & $0.16,0.15,0.12$ & $0.007,0.010,0$ & $5 \times 10^{-4}, 6 \times 10^{-4}, 0$ \\
\hline & $\mathrm{GC}$ & $0.006,0.007,0.005$ & $3 \times 10^{-4}, 5 \times 10^{-4}, 0$ & $9 \times 10^{-7}, 0,0$ \\
\hline & YSC & $10^{-4}, 2 \times 10^{-4}, 0$ & $6 \times 10^{-6}, 9 \times 10^{-6}, 0$ & $0,0,0$ \\
\hline \multirow[t]{3}{*}{ LONG_DELAY } & NSC & $0.16,0.15,0.14$ & $0.007,0.010,0$ & $4 \times 10^{-4}, 6 \times 10^{-4}, 0$ \\
\hline & GC & $0.006,0.007,0.003$ & $3 \times 10^{-4}, 5 \times 10^{-4}, 0$ & $0,0,0$ \\
\hline & YSC & $10^{-4}, 2 \times 10^{-4}, 0$ & $7 \times 10^{-6}, 10^{-5}, 0$ & $0,0,0$ \\
\hline \multirow[t]{3}{*}{ BROAD_VESC } & NSC & $0.20,0.20,0.16$ & $0.012,0.015,0$ & $0.004,0.005,0$ \\
\hline & $\mathrm{GC}$ & $0.012,0.013,0.003$ & $6 \times 10^{-4}, 9 \times 10^{-4}, 0$ & $4 \times 10^{-6}, 8 \times 10^{-6}, 0$ \\
\hline & YSC & $4 \times 10^{-4}, 5 \times 10^{-4}, 0$ & $3 \times 10^{-5}, 3 \times 10^{-5}, 0$ & $0,0,0$ \\
\hline \multirow[t]{3}{*}{ NARROW_VESC } & NSC & $0.14,0.13,0.08$ & $0.006,0.007,0$ & $10^{-4}, \times 10^{-4}, 0$ \\
\hline & $\mathrm{GC}$ & $0.003,0.005,0$ & $2 \times 10^{-4}, 3 \times 10^{-4}, 0$ & $0,0,0$ \\
\hline & YSC & $8 \times 10^{-5}, 10^{-4}, 0$ & $7 \times 10^{-6}, 4 \times 10^{-6}, 0$ & $0,0,0$ \\
\hline
\end{tabular}

Column 1: Name of the model. Column 2: Star cluster type (NSC, GC or YSC). Column 3: Fraction of $N g$ BBHs $f_{>1 g}$. The three values reported in each line refer to $Z=0.0002,0.002$ and 0.02 . Column 4 : Fraction of BBHs with primary mass in the pair instability gap $f_{\text {PISN }}$. The three values reported in each line refer to $Z=0.0002,0.002$ and 0.02 . Column 5: Fraction of IMBH mergers $f_{\mathrm{IMBH}}$. The three values reported in each line refer to $Z=0.0002,0.002$ and 0.02 .

\subsection{BHs in the Mass Gap and IMBHs}

Hierarchical mergers could be responsible for the formation of BHs with mass in the pair instability mass gap $\left(\sim 60-120 \mathrm{M}_{\odot}\right)$ or even in the IMBH regime $\left(>100 \mathrm{M}_{\odot}\right)$. The bottom left panel of Figure 7 shows $f_{\text {PISN }}$ defined as $f_{\text {PISN }}=N_{\text {PISN }} / N_{\mathrm{BBH}}$, where $N_{\text {PISN }}$ is the number of $\mathrm{BBH}$ mergers with primary mass in the pair instability mass gap, while $N_{\mathrm{BBH}}$ is the number of all BBH mergers. In our fiducial model and in NSCs, $\sim 0.7 \%$ of all BBH mergers contain at least one $\mathrm{BH}$ in the pair instability mass gap at the lowest metallicity $(Z=0.0002)$. This percentage decreases as metallicity increases and drops to zero at $Z \geq 0.012$. The other models follow the same trend with metallicity. The HIGH_MASS model is the one with the largest value of $f_{\mathrm{PISN}}$ : In this case, up to $7.5 \%$ of all the BBH mergers contain at least one $\mathrm{BH}$ in the pair instability mass gap at the lowest metallicity $(Z=0.0002)$. These percentages should be lowered by a factor of $\gtrsim 10$ in GCs and by a factor of $\sim 10^{3}$ in YSCs. 

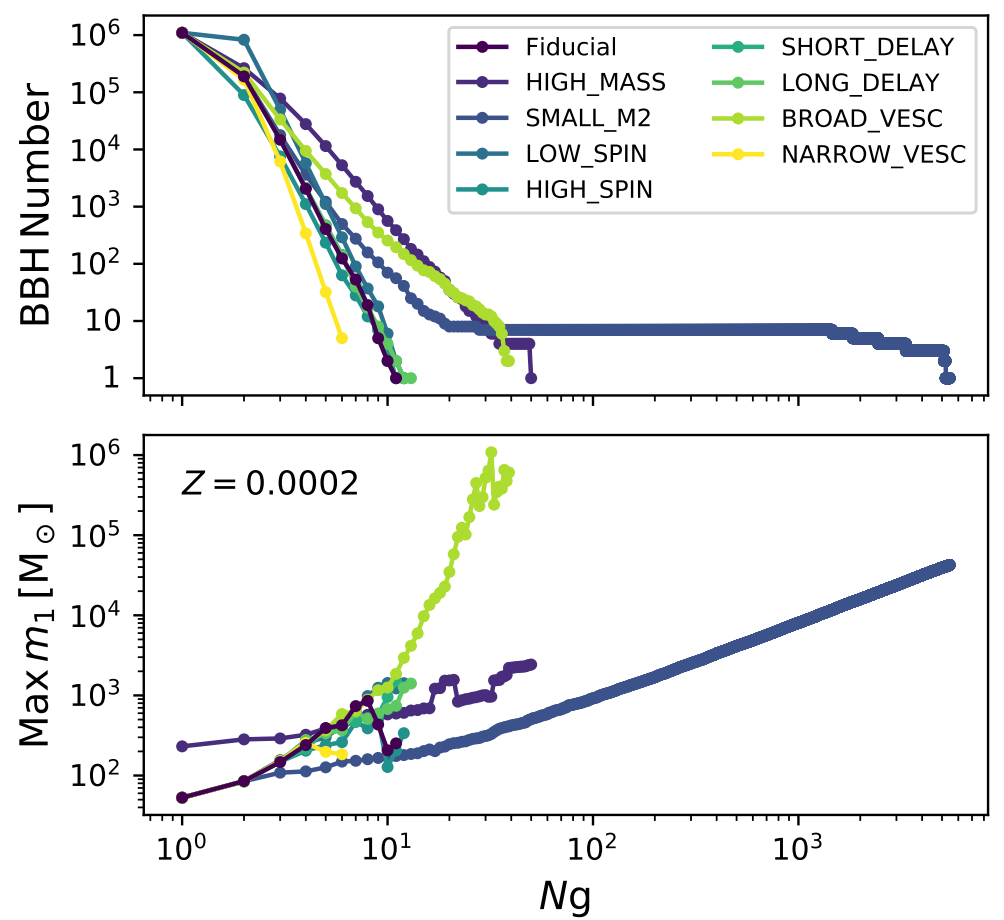

Figure 6. Top: number of BBHs in each generation as a function of the generation number $\mathrm{Ng}$ for hierarchical BBHs in NSCs with metallicity $Z=0.0002$. Bottom: Maximum primary BH mass in each generation as a function of the generation number $\mathrm{Ng}$ for hierarchical BBHs in NSCs with metallicity $Z=0.0002$. We show models A5F05 (fiducial), HIGH_MASS, SMALL_M2, LOW_SPIN, HIGH_SPIN, SHORT_DELAY, LONG_DELAY, BROAD_VESC, and NARROW_VESC.
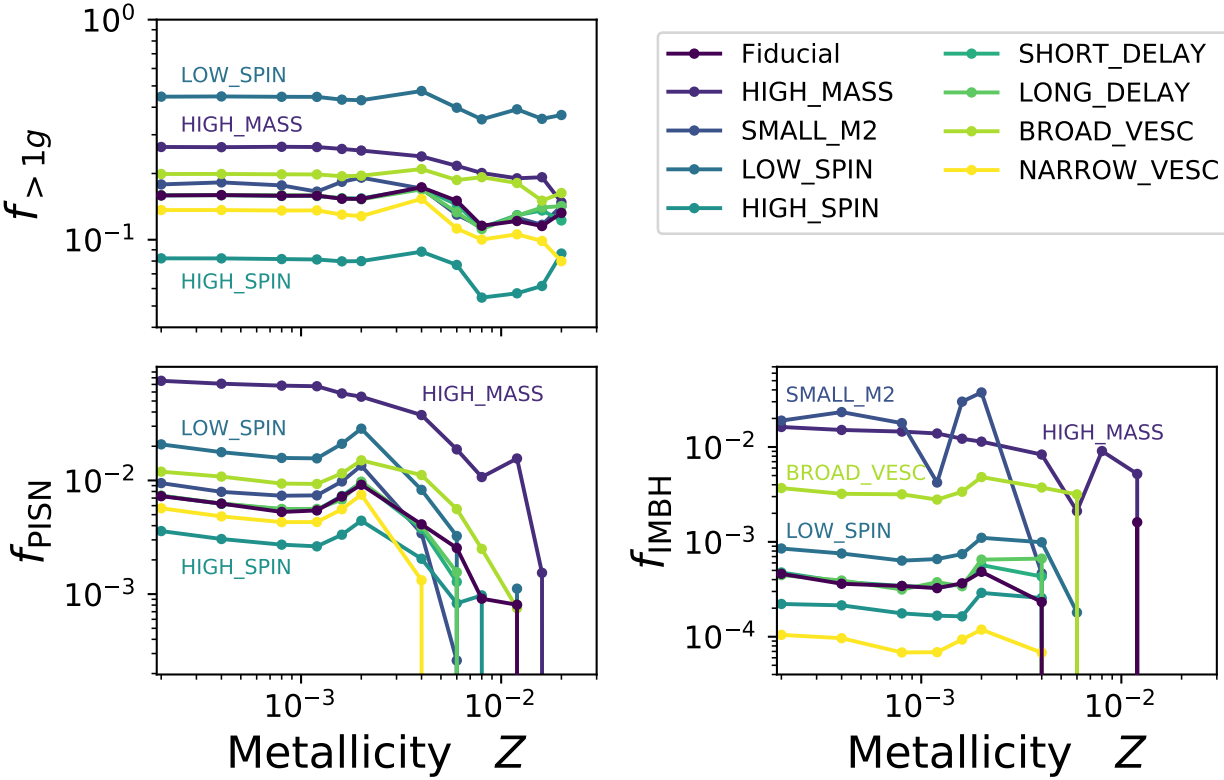

Figure 7. Top: $f_{>1 g}$ is the number of $N g$ BBH mergers with $N>1$ divided by the total number of BBH mergers. Bottom left: $f_{\text {PISN }}$ is the number of $\mathrm{Ng}$ BBH mergers with a primary mass in the pair instability mass gap $\left(m_{1} \in[60,120] \mathrm{M}_{\odot}\right)$, divided by the total number of $\mathrm{BBH}$ mergers. Bottom right: $f_{\mathrm{IMBH}}$ is the number of $\mathrm{Ng} \mathrm{BBH}$ mergers with a primary mass in the IMBH regime $\left(m_{1} \geq 100 \mathrm{M}_{\odot}\right)$, divided by the total number of BBH mergers. $f_{>1}, f_{\text {PISN }}$ and $f_{\text {IMBH }}$ refer to NSCs only and are shown as a function of the metallicity Z. We show models A5F05 (fiducial), HIGH_MASS, SMALL_M2, LOW_SPIN, HIGH_SPIN, SHORT_DELAY, LONG_DELAY, BROAD_VESC, and NARROW_VESC. 
In the bottom right panel of Figure 7, we show the fraction of IMBH mergers $f_{\mathrm{IMBH}}$, defined as $f_{\mathrm{IMBH}}=N_{\mathrm{IMBH}} / N_{\mathrm{BBH}}$, where $N_{\mathrm{IMBH}}$ is the number of $\mathrm{BBH}$ mergers with primary mass $m_{1}>10^{2} \mathrm{M}_{\odot}$. The fraction of IMBH mergers follows the same trend with metallicity as $f_{\text {PISN }}$ : It is higher at lower $Z$ and drops to zero at $Z \geq 4 \times 10^{-3}$. In the fiducial model, $f_{\text {IMBH }} \sim 5 \times 10^{-4}$ at $Z=0.0002$ in NSCs. We find no IMBHs in GCs and YSCs in the fiducial case. The fraction of IMBH mergers is maximum in the SMALL_M2 simulation, where $f_{\text {IMBH }} \sim 2 \times 10^{-2}$ at $Z=0.0002$. Moreover, $f_{\text {IMBH }} \sim 6 \times 10^{-4}$ and $\sim 3 \times 10^{-5}$ at $Z=0.0002$ in the HIGH_MASS case for GCs and YSCs, respectively. Table 2 reports the values of $f_{\mathrm{PISN}}$ and $f_{\mathrm{IMBH}}$ in detail.

\subsection{Merger Rates}

Figures 8 and 9 show the merger rate density evolution for all our models, calculated as detailed in Section 2.7. The contribution of each channel to the total merger rate density is set by the value of $f_{i}(z)$ because hierarchical mergers are only a small fraction of the total BBH mergers (Figure 7). Since $f_{i}(z)$ is highly uncertain, the relative importance of different channels in Figures 8 and 9 can change wildly and is only indicative. The uncertainty is particularly large for field and YSCs.
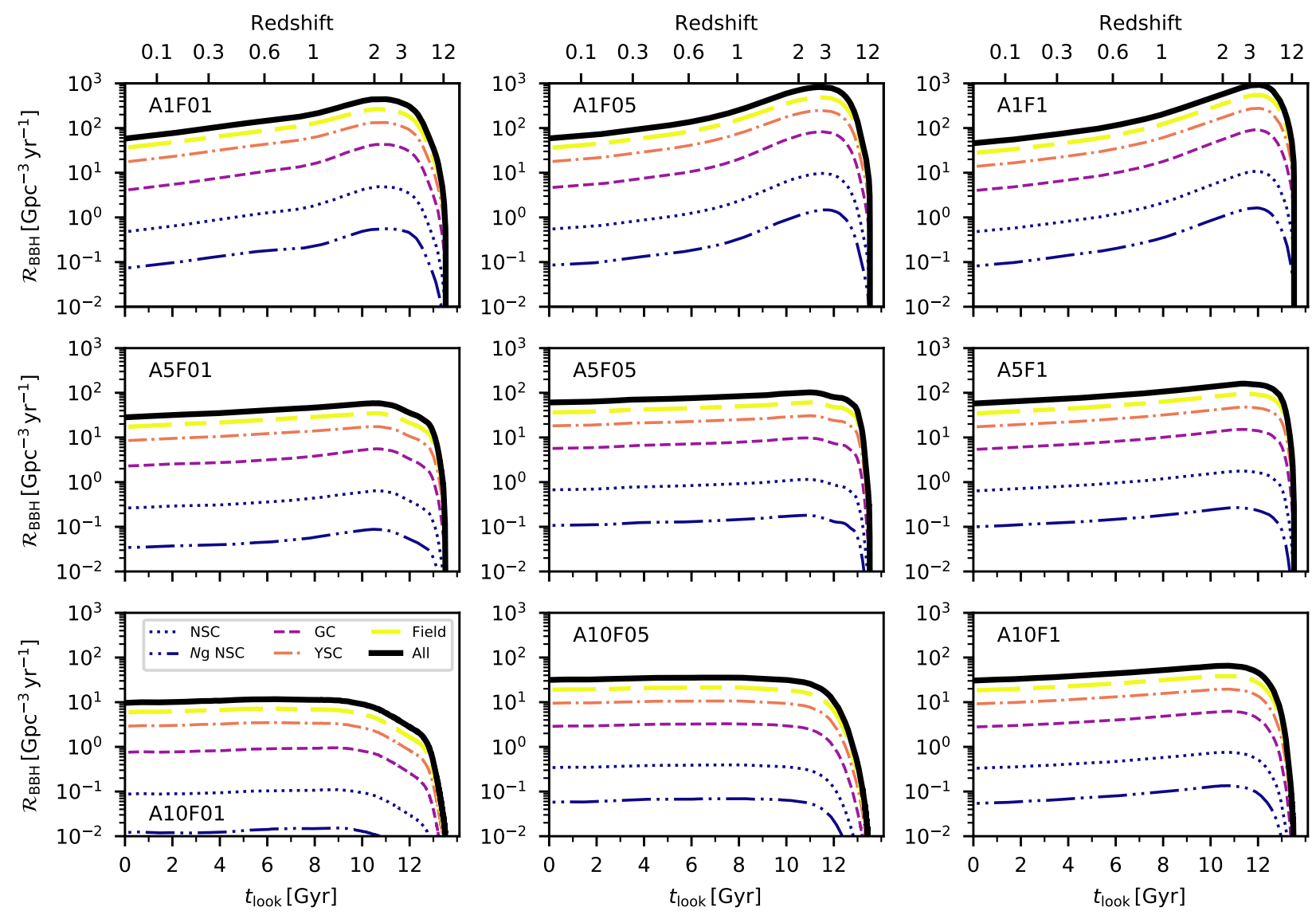

Figure 8. From top to bottom and from left to right: $\mathrm{BBH}$ merger rate density in the comoving frame as a function of look-back time (bottom $x$-axis) and redshift (top $x$-axis) for the simulations A1F01, A1F05, A1F1, A5F01, A5F05, A5F1, A10F01, A10F05, and A10F1. Black thick line: Total merger rate density; yellow long-short dashed line: BBH merger rate density from field binaries; pink dot-dashed line: $\mathrm{BBH}$ merger rate density from YSCs; violet dashed line: $\mathrm{BBH}$ merger rate density from GCs; blue dotted line: BBH merger rate density from NSCs; blue dot-dot-dashed line: BBH merger rate density from NSCs if we consider only $\mathrm{Ng}$ BBHs. 


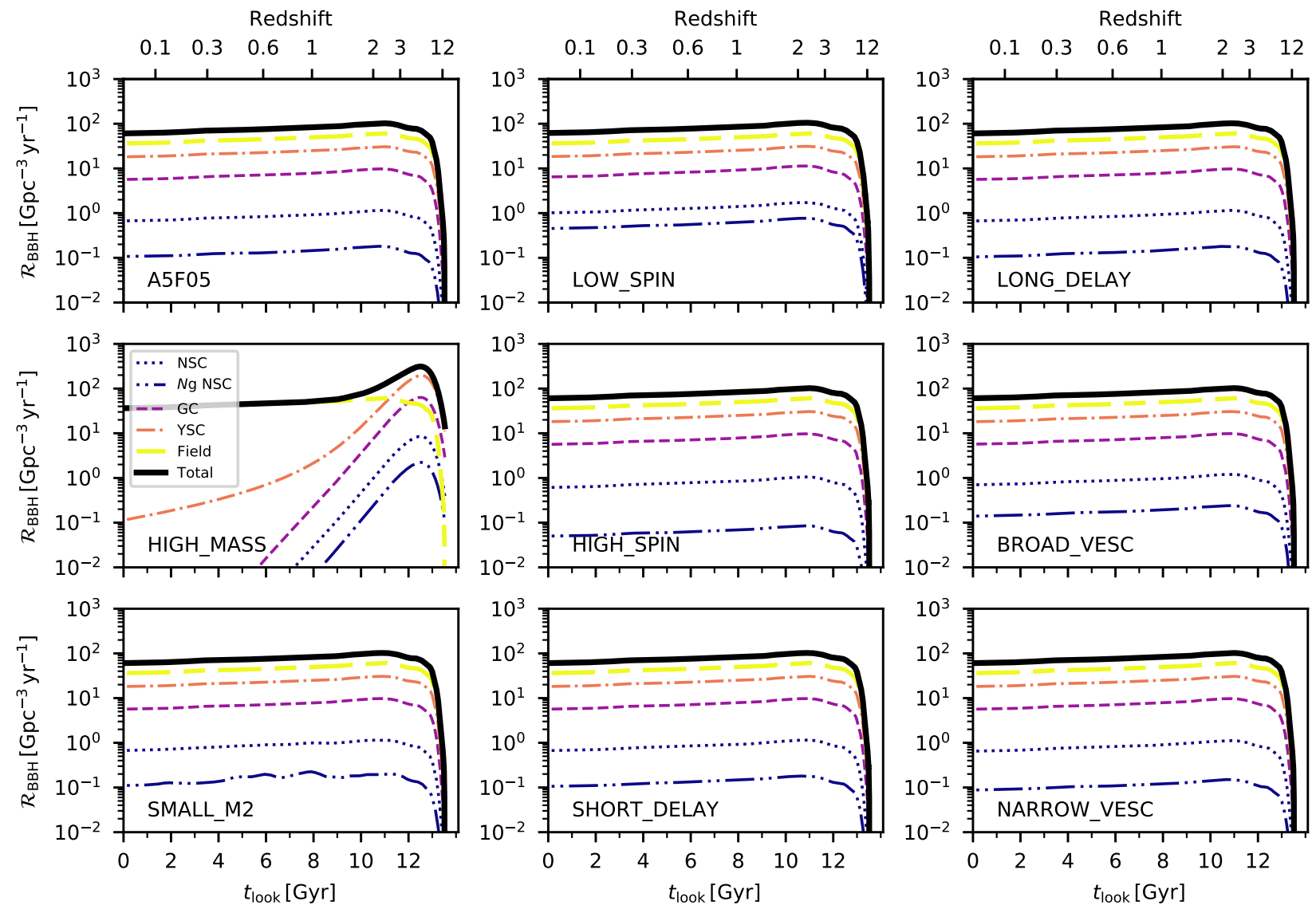

Figure 9. Same as Figure 8 but for the simulations A5F05 (fiducial), LOW_SPIN, LONG_DELAY, HIGH_MASS, HIGH_SPIN, BROAD_VESC,SMALL_M2,SHORT_DELAY, and NARROW_VESC.

Models with $\alpha=1$ have a higher merger rate than models with $\alpha=5,10$. The merger rate evolution of dynamical BBHs in the HIGH_MASS case is remarkably different from the other cases. The reason is our choice of the delay time distribution of $1 \mathrm{~g}$ BBHs $\left(d N / d t \propto t^{-1}\right)$, which does not take into account a possible dependence of $t_{\text {delay }}$ on the mass and other properties of BBHs. In particular, the delay time distribution obtained with MOBSE tends to deviate from the $d N / d t \propto t^{-1}$ trend when $t_{\text {delay }}<1$ Gyr. Hence, dynamical BBHs in the HIGH_MASS case have shorter delay times than the fiducial case.

Figures 8 and 9 also show the BBH merger rate density we obtain if we consider only $\mathrm{Ng}$ BBHs in NSCs. In the local Universe, the merger rate density of $\mathrm{Ng}$ BBHs in NSCs ranges from $\sim 10^{-2}$ to $\sim 0.2 \mathrm{Gpc}^{-3} \mathrm{yr}^{-1}$. For GCs and YSCs we obtain lower values because, even if these star clusters are likely more common than NSCs, the occurrence of $\mathrm{Ng} \mathrm{BBH}$ mergers in GCs and YSCs is lower than in NSCs (e.g., Section 3.1).

\subsection{Mass Distribution at Different Redshifts}

Figure 10 shows the total mass distribution of primary BHs in the source frame at redshift $z=1$. NSCs are responsible for the high mass tail $\left(m_{1} \gtrsim 100 \mathrm{M}_{\odot}\right)$ at all redshifts and in all models. We show only the distribution at $z=1$ because we do not see significant changes of the mass distribution with redshift in all cases but the HIGH_MASS model. In this case, the importance of dynamical BBHs drops at redshift zero because of the different delay time distributions (Figure 9). 

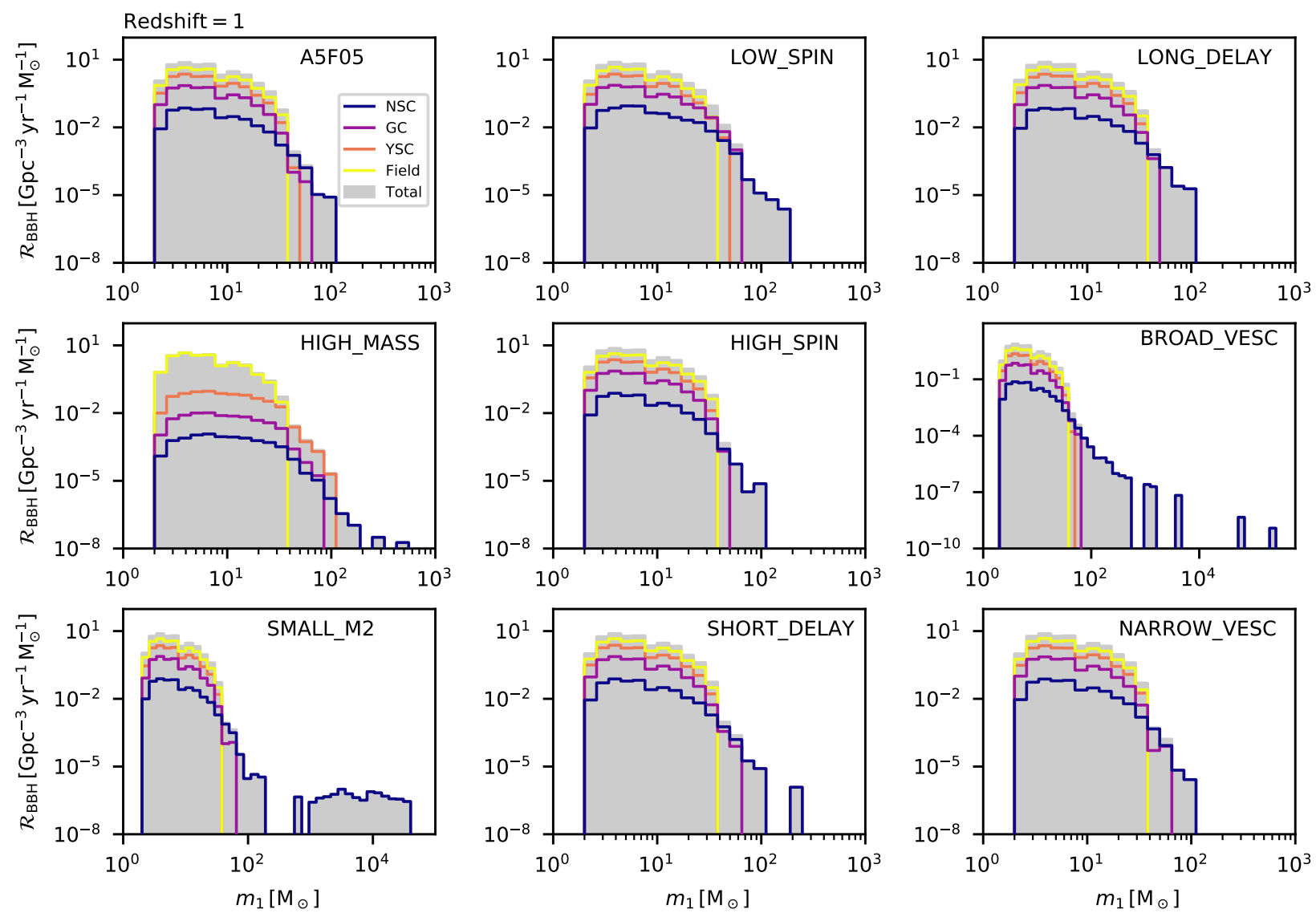

Figure 10. From top to bottom and from left to right: Merger rate density per unit primary mass as a function of BBH mass at redshift $z=1$ for the simulations A5F05 (fiducial), LOW_SPIN, LONG_DELAY, HIGH_MASS, HIGH_SPIN, BROAD_VESC, SMALL_M2, SHORT_DELAY, and NARROW_VESC. Grey histogram: Sum of all formation channels; yellow: Field binaries; pink: YSCs; violet: GCs; blue: NSCs.

Figure 11 shows the mass distribution of primary BHs at redshift $z=2$ for NSCs only. We separate $1 \mathrm{~g}$ BBHs from $\mathrm{Ng}$ BBHs with $N>1$. The maximum mass of $1 \mathrm{~g}$ BBHs extends up to $\sim 40 \mathrm{M}_{\odot}$ in all simulations but the HIGH_MASS case. In the HIGH_MASS case, $1 \mathrm{~g}$ BHs with mass up to $\sim 100 \mathrm{M}_{\odot}$ are possible because this model includes BHs that form with mass in the pair instability gap from the merger of massive stars [56] and acquire companions by dynamical exchanges.

The mass of $\mathrm{Ng}$ BHs extends up to $\sim 100-200 \mathrm{M}_{\odot}$ in most models, with the exception of the following runs. In the HIGH_MASS case, we find primary BHs with mass up to $\sim 600-10^{3} \mathrm{M}_{\odot}$. In the SMALL_M2 case, the most massive BH reaches $\sim 5 \times 10^{4} \mathrm{M}_{\odot}$. Finally, this realization of the BROAD_VESC model produces one single $\mathrm{BH}$ with mass $\sim 4.3 \times 10^{5} \mathrm{M}_{\odot}$. To obtain the shown distributions, we started from catalogs of $\geq 10^{6} \mathrm{BBHs}$. Figure 11 confirms that the distribution of $\mathrm{Ng}$ BBHs strongly depends not only on the properties of the environment (e.g., $v_{\text {esc }}$ ) but also on the mass distribution of $1 \mathrm{~g} \mathrm{BHs}$. 

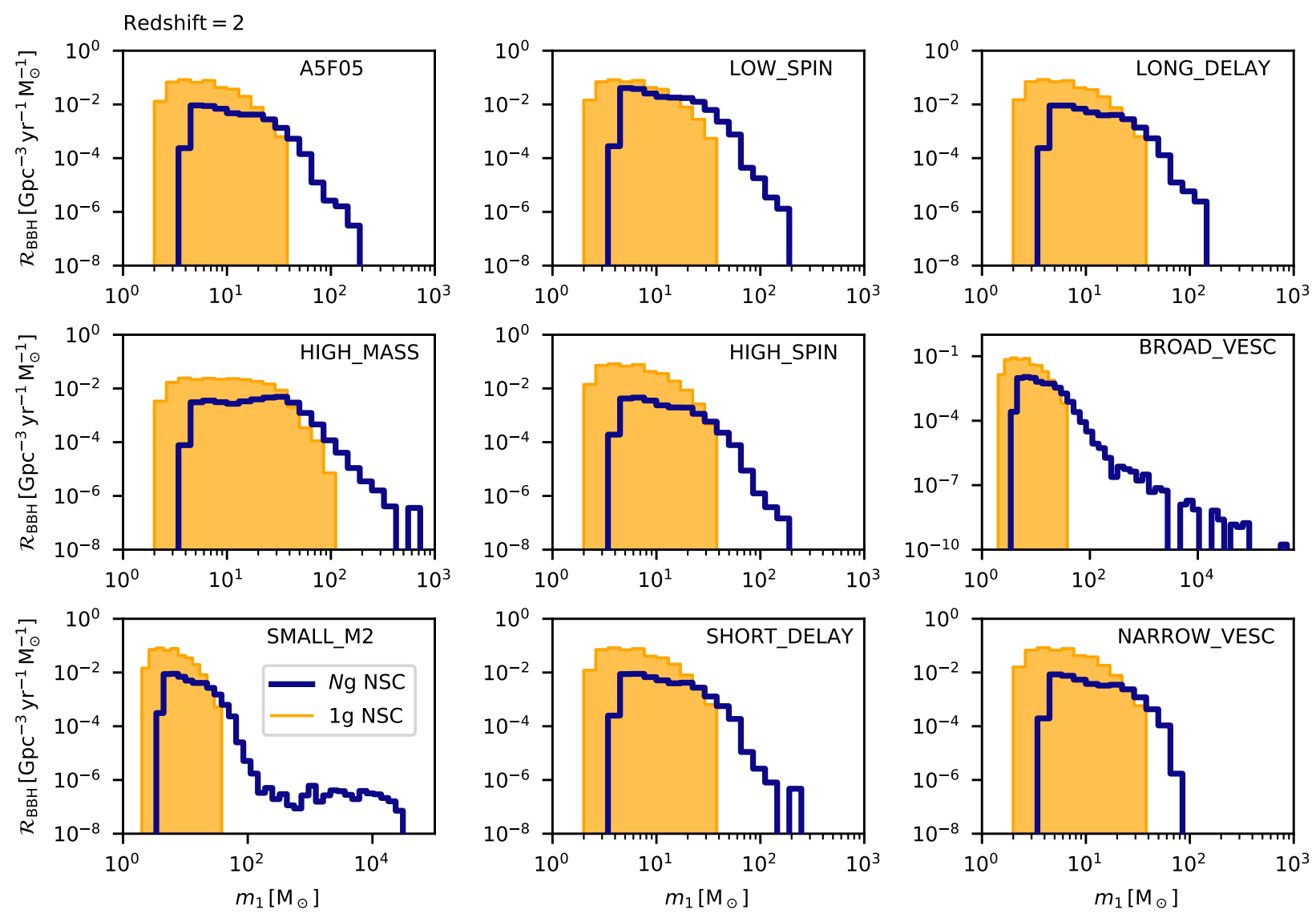

Figure 11. Merger rate density per unit primary mass as a function of BBH mass at redshift $z=2$ for BBHs in NSCs. Filled orange histogram: $1 \mathrm{~g}$ BBHs; blue histogram: $\mathrm{Ng}$ BBHs. From top to bottom and from left to right: Simulations A5F05 (fiducial), LOW_SPIN, LONG_DELAY, HIGH_MASS, HIGH_SPIN, BROAD_VESC, SMALL_M2, SHORT_DELAY, and NARROW_VESC.

\subsection{Comparison with BBHs in GWTC-2}

To compare our models against GW events in the first (O1), second (O2) and in the first part of the third observing run (O3a) of the LIGO-Virgo collaboration (hereafter, the GWTC-2 catalog [9]), we use a hierarchical Bayesian approach. In this framework, the posterior for a set of data $\{h\}^{k}$ observed during an observation time $T_{o b s}$ and a model parametrized by $\lambda$ is well described by an in-homogeneous Poisson process $[187,188]$

$$
p\left(\lambda, N_{\lambda} \mid\{h\}^{k}\right) \sim \mathrm{e}^{-\mu_{\lambda}} \pi\left(\lambda, N_{\lambda}\right) \prod_{k=1}^{N_{o b s}} N_{\lambda} \int_{\theta} \mathcal{L}^{k}\left(\{h\}^{k} \mid \theta\right) p(\theta \mid \lambda) \mathrm{d} \theta,
$$

where $\theta$ are the GW parameters, $N_{\lambda}$ is the number of events predicted by the astrophysical model, $\mu_{\lambda}$ is the predicted number of detections associated with the model and GW detector, $\pi\left(\lambda, N_{\lambda}\right)$ is the prior distribution on $\lambda$ and $N_{\lambda}$, and $\mathcal{L}^{k}\left(\{h\}^{k} \mid \theta\right)$ is the likelihood of the $k$-th detection.

The predicted number of detections is given by $\mu(\lambda)=N_{\lambda} \beta(\lambda)$, where $\beta(\lambda)=\int_{\theta} p(\theta \mid \lambda) p_{\operatorname{det}}(\theta) \mathrm{d} \theta$ is the detection efficiency of the model; $p_{\operatorname{det}}(\theta)$ is the probability of detecting a source with parameters $\theta$ and can be inferred by computing the optimal signal-to-noise ratio and comparing it to a detection threshold [189]. The values for the event's log-likelihood are derived from the posterior and prior samples released by 
the LIGO-Virgo collaboration, such that the integral in the above equation is approximated with a Monte Carlo approach as

$$
\int_{\theta} \mathcal{L}^{k}\left(\{h\}^{k} \mid \theta\right) p(\theta \mid \lambda) \mathrm{d} \theta \sim \frac{1}{N_{s}^{k}} \sum_{i=1}^{N_{s}^{k}} \frac{p\left(\theta_{i}^{k} \mid \lambda\right)}{\pi^{k}\left(\theta_{i}^{k}\right)}
$$

where $\theta_{i}^{k}$ is the $i$-th posterior sample for the $k$-th detection and $N_{s}^{k}$ is the total number of posterior samples for the $k$-th detection. To compute the prior term in the denominator, we use a Gaussian kernel density estimation.

In practice, for each model, we generate a catalog of a fixed number of sources (fixed to 50,000 sources), such that the sources are distributed according to the merger rate density of the model. Each entry of the catalog is represented by a set of parameters $\theta=\left\{\mathcal{M}_{c}, q, \chi_{\text {eff }}, z\right\}$, where $\mathcal{M}_{c}$ is the chirp mass of the source, $q$ the mass ratio, $\chi_{\text {eff }}$ is the effective spin and $z$ the redshift, that was set to take values between 0 and 2. More details on this procedure are described in $[188,189]$.

In our analysis, our model distribution is the sum of the contributions from multiple channels (isolated BBHs, dynamical BBHs in YSCs, GCs and NSCs) weighted by mixing fraction hyper-parameters as

$$
p\left(\theta \mid \xi_{1}, \xi_{2}, \xi_{3}, \xi_{4}, \lambda\right)=\xi_{1} p(\theta \mid \text { Field }, \lambda)+\xi_{2} p(\theta \mid \text { YSC }, \lambda)+\xi_{3} p(\theta \mid \text { GC, } \lambda)+\xi_{4} p(\theta \mid \text { NSC, } \lambda),
$$

where $\xi_{1}, \xi_{2}, \xi_{3}$ and $\xi_{4}$ are the mixing fractions of the field (Field), young star cluster (YSC), globular cluster (GC) and nuclear star cluster (NSC) scenarios, defined so that $\xi_{1}+\xi_{2}+\xi_{3}+\xi_{4}=1$. Based on this definition, the mixing fraction for each channel is the fraction of merger events associated with that specific channel.

Figure 12 shows the posterior probability distribution of the mixing fractions for the four different channels and for a selection of our models. The results show large variations from one model to another. The strong fluctuations of $\xi_{i}$ from one model to another indicate that the mixing fractions are extremely sensitive to the hyper-parameters $\lambda$. Considering the large uncertainties on the astrophysical models, different assumptions about the $1 \mathrm{~g}$ BBH mass function or other key parameters deeply affect the values of $\tilde{\xi}_{i}$. However, there is one common feature: The results of GWTC-2 support the co-existence of multiple channels. In fact, the median value of the mixing fraction is significantly higher than zero for at least two of the four channels in each specific model. For example, the posterior distributions of the mixing fraction of the NSC, YSC and Field channels peak at values significantly larger than zero in the A5F05, LONG_DELAY, SHORT_DELAY, HIGH_SPIN, BROAD_VESC, and NARROW_VESC models. This result is in agreement with previous work [189-192].

Overall, the Field model seems to be associated with the higher values of the mixing fraction, with median values between $\xi_{1} \sim 0.3$ (HIGH_SPIN model) and $\xi_{1} \sim 0.85$ (HIGH_MASS model). The rates and the mass function are two key ingredients here: The predicted rates are higher for the Field than for the other channels in all our models and the mass function of Field BBHs has a preference for low values; the BBH population inferred from GWTC-2, after correcting for detection biases, is better represented by a mixture model in which Field binaries give a substantial contribution. The HIGH_SPIN case is the one that maximally "penalizes" the Field case because of an excess of large positive values of $\chi_{\text {eff }}$ in this channel. This is the main reason why YSCs and NSCs are expected to contribute significantly to the overall population in this specific model. 

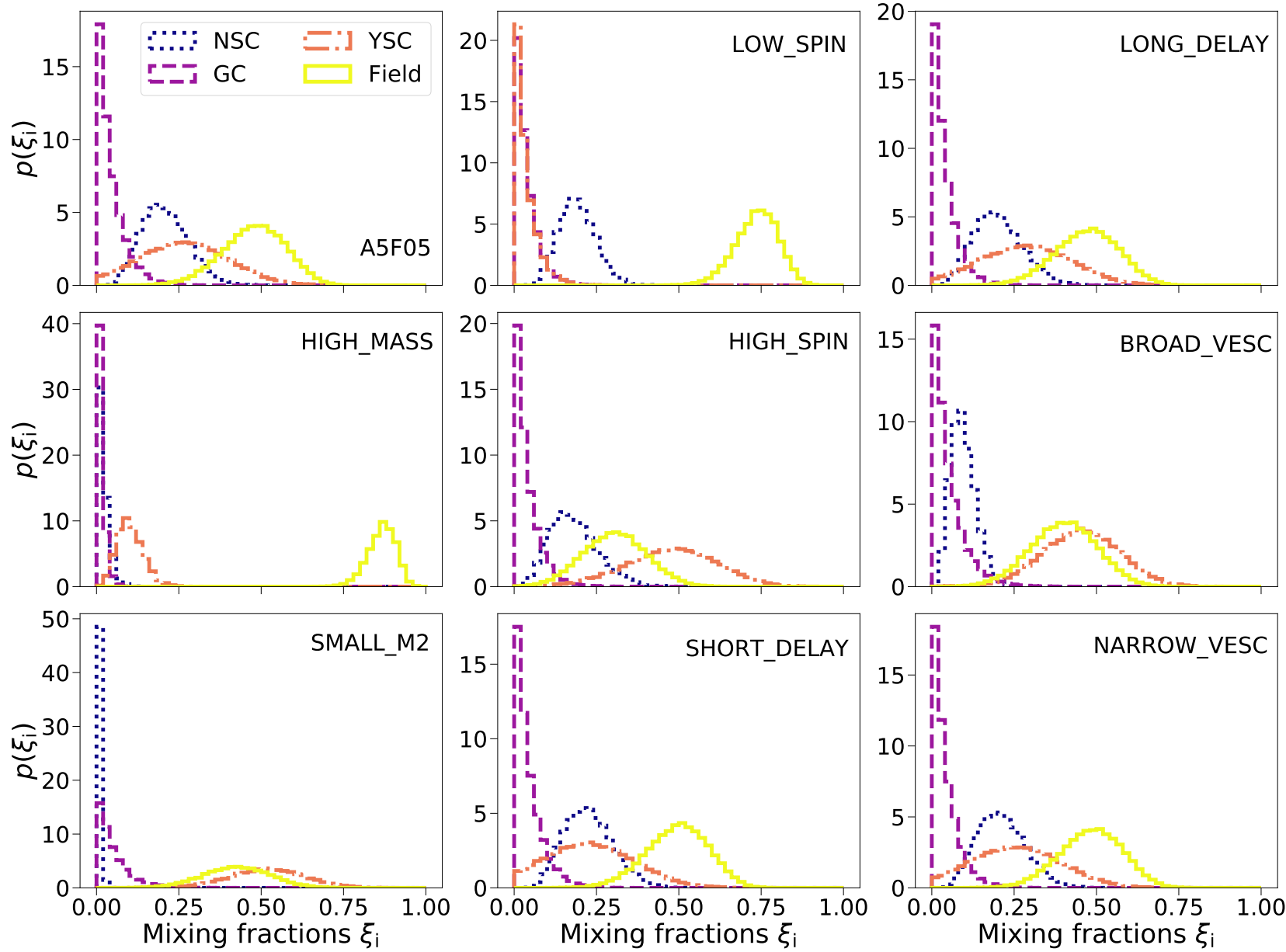

Figure 12. Posterior probability distribution of the mixing fractions $\xi_{\mathrm{i}}$ for our multi-channel analysis. The mixing fractions $\xi_{\mathrm{i}}$, where $\mathrm{i}=1,2,3$ and 4 for Field, YSCs, GCs and NSCs, are defined in Equation (18). From top to bottom and from left to right: Simulations A5F05 (fiducial), LOW_SPIN, LONG_DELAY, HIGH_MASS, HIGH_SPIN, BROAD_VESC,SMALL_M2, SHORT_DELAY, and NARROW_VESC.

In most models, GCs are associated with low mixing fractions. This mostly happens because NSCs "work" better than GCs to explain the most massive events (like GW190521) and thus are preferred by our formalism. In the SMALL_M2 case, both GCs and NSCs are associated with low mixing fractions because they tend to predict too many massive primary BHs with very low mass ratios.

The details of these results might strongly depend on the star formation rate and metallicity evolution model, which can deeply change the merger rate $[36,38,193,194]$. We will investigate the impact of these quantities in a follow-up study.

\section{Discussion of the Main Caveats}

We presented a new model that can be used to rapidly simulate hierarchical mergers in different environments (NSCs, GCs and YSCs), exploring a broad parameter space (e.g., progenitor's metallicity, binary evolution parameters such as $\alpha$ and $f_{\mathrm{MT}}$, escape velocity from the parent star cluster, delay times and $1 \mathrm{~g}$ spin distribution). The treatment of dynamical pairing of $\mathrm{Ng} \mathrm{BBHs}$ is still approximate: We assume that the retained merger remnants find a new companion and merge over a timescale $d N / d t \propto t^{-1}$. This is in agreement with the findings of [102], but could be improved with an analytic treatment of dynamical hardening [115]. Furthermore, we assume that BHs can only be ejected by relativistic kicks, i.e., neglect dynamical recoil via close encounters. Finally, we assume that the star cluster does not evolve with time: It has a constant escape velocity. As shown in 
previous work [195-198], the properties of the star cluster might significantly change with time and the growth of an IMBH is strongly linked to the evolution of the host star cluster. For example, if we assume constant cluster mass, the half-mass ratio is expected to grow as $r_{\mathrm{h}} \propto t^{2 / 3}$ and the escape velocity to decrease with time as $v_{\mathrm{esc}} \propto t^{-1 / 3}$ [199]. These two effects might slow down or even suppress the growth of an IMBH in the late evolutionary stages [102].

In our fiducial model, we assume that the stellar binaries which give birth to $1 \mathrm{~g}$ BHs are primordial binaries and are not ionized by dynamical interactions. This assumption is motivated by the properties of such binaries. A BBH merger progenitor has an initial binding energy

$$
E_{b} \sim 6 \times 10^{49} \mathrm{erg} \mathrm{s}^{-1}\left(\frac{m_{1}}{50 \mathrm{M}_{\odot}}\right)\left(\frac{m_{2}}{50 \mathrm{M}_{\odot}}\right)\left(\frac{1000 \mathrm{R}_{\odot}}{\mathcal{A}}\right),
$$

where $\mathcal{A}$ is the initial semi-major axis. The typical kinetic energy of a star in a star cluster is

$$
E_{K} \sim 10^{47} \mathrm{erg} \mathrm{s}^{-1}\left(\frac{\langle m\rangle}{1 \mathrm{M}_{\odot}}\right)\left(\frac{\sigma_{\mathrm{SC}}}{100 \mathrm{~km} \mathrm{~s}^{-1}}\right)^{2}
$$

where $\langle m\rangle$ is the average stellar mass in the cluster and $\sigma_{\mathrm{SC}}$ is the velocity dispersion. In the example, we consider an extremely high velocity dispersion $\sigma_{\mathrm{SC}}=100 \mathrm{~km} \mathrm{~s}^{-1}$. Hence, binaries that will produce $\mathrm{BBH}$ mergers are hard binaries even in the most extreme star clusters and should survive ionization. This assumption breaks in the immediate vicinity of a super-massive $\mathrm{BH}$. For example, inside the influence radius of a supermassive $\mathrm{BH}$ with mass $m_{\mathrm{BH}}=10^{6} \mathrm{M}_{\odot}$, the typical velocities are $\sim 120 \mathrm{~km} \mathrm{~s}^{-1}(a / 0.01 \mathrm{pc})^{-1 / 2}\left(m_{\mathrm{BH}} / 10^{6} \mathrm{M}_{\odot}\right)$. In this extreme case, even BBHs and their stellar progenitors might be soft binaries and might be broken. On the other hand, dynamical hardening might also be very effective as the $\mathrm{BBH}$ gets closer to a supermassive $\mathrm{BH}$ by dynamical friction, allowing the $\mathrm{BBH}$ to avoid ionization and even speeding up its merger [77].

Here, we make no assumptions about the formation of NSCs. If some of them, if not all, are formed by the hierarchical assembly of GCs $[169,170]$, this might have a crucial impact on the population of BBHs. In fact, the GCs might already be depleted of merger remnants (because of their relatively low escape velocity) before merging to build up a NSC. Moreover, we neglect the AGN disk formation channel [78-81,200]. Including the physics of AGN disks can boost the contribution of galactic nuclei to the total merger rate and to $\mathrm{Ng}$ mergers. AGN disk physics can further speed up the pairing and merger of our BHs. We will include the AGN disk scenario in future work.

Arca Sedda et al. (2020, [76]) found remnant masses only up to $\sim 200 \mathrm{M}_{\odot}$, significantly lower than the results presented here for most models. The main reason for this difference is that [76] fixed the escape velocity from NSCs to $v_{\mathrm{esc}}=100 \mathrm{~km} \mathrm{~s}^{-1}$ and did not change this parameter. Our results are consistent with other models (e.g., [102]), where higher values of $v_{\text {esc }}$ are explored. This result is remarkable when considering that [102] adopt a more accurate model for dynamical interactions than the one presented here. Hence, escape velocities are the key ingredient to understand the mass spectrum of BHs in NSCs.

Finally, we include a simple redshift dependence based on the $f_{i}(t)$ functions. Alternative redshift dependencies can be obtained by changing $f_{i}(t)$. For example, if we assume $f_{\mathrm{NSC}}=0.1$ (constant with redshift), we obtain an upper limit to the merger rate density associated with NSCs because they are unlikely to contribute to $10 \%$ of the overall cosmic star formation rate. Under such extreme assumption, the local $\mathrm{BBH}$ merger rate density from NSCs is $\mathcal{R}_{\mathrm{NSC}} \approx 7-10 \mathrm{Gpc}^{-3} \mathrm{yr}^{-1}$, i.e., approximately a factor of 10 higher than the models we presented in Figures 8 and 9.

\section{Summary}

Hierarchical mergers in dynamical environments can lead to the formation of BHs with mass higher than the limits imposed by pair instability, core-collapse supernovae and 
stellar evolution theory. Here, we presented a fast semi-analytic method to draw the main properties (masses, spins, merger rate) of hierarchical BBHs, while probing the relevant parameter space.

In our models, NSCs are the dominant environment for the formation of hierarchical BBHs. In our fiducial model (A5F05), primary BHs with mass up to $\sim 10^{3} \mathrm{M}_{\odot}$ can form in NSCs, while the maximum primary BH mass is $\sim 100 \mathrm{M}_{\odot}$ for both GCs and YSCs.

We find that the mass distribution of $1 \mathrm{~g}$ BBHs has a crucial impact on the mass distribution of $\mathrm{Ng}$ BHs with $N>1$. The metallicity of the progenitor is a key ingredient to shape the distribution of $\mathrm{Ng}$ BBHs because it affects both the number and the maximum mass of BBHs. The common envelope $\alpha$ parameter and the accretion efficiency $f_{\mathrm{MT}}$ also play a role, with smaller values of $\alpha$ leading to higher merger rates and higher values of $f_{\mathrm{MT}}$ leading to more top-heavy $\mathrm{BH}$ mass functions.

If BHs with mass in the pair instability gap are allowed to form by stellar mergers [56], the mass distribution of $\mathrm{Ng}$ BBHs is skewed toward significantly larger masses (HIGH_MASS model). Primary BH masses up to a few $\times 10^{4} \mathrm{M}_{\odot}$ can be obtained in NSCs if only $\mathrm{Ng}-1 \mathrm{~g}$ mergers are allowed to take place, i.e., if we prevent the secondary $\mathrm{BH}$ from being a merger remnant itself (SMALL_M2 model). The main reason is that relativistic kicks are smaller if the mass ration $q=m_{2} / m_{1}$ tends to zero.

The escape velocity of the parent star cluster $\left(v_{\mathrm{esc}}\right)$ is probably the most important parameter to set the maximum $\mathrm{BH}$ mass. If we assume that the distribution of escape velocities from NSCs is $\log _{10}\left(v_{\mathrm{esc}} / \mathrm{km} \mathrm{s}^{-1}\right)=2 \pm 0.3$ (BROAD_VESC model), BHs with mass up to $\sim 10^{6} \mathrm{M}_{\odot}$ are allowed to form in the NSCs with the highest escape velocities. This result is consistent with $[47,102]$.

While BBHs in GCs and YSCs do not exceed the 5th and the 3rd generation, respectively, we expect at least 10 different $\mathrm{BBH}$ generations in NSCs. This number grows up to a few thousands if $\mathrm{Ng}-1 \mathrm{~g} \mathrm{BBH}$ are the only way to produce hierarchical mergers (SMALL_M2 model).

In our fiducial model, the fraction of $\mathrm{Ng}$ BBHs is $f_{>1 g} \sim 0.15$ in NSCs, which lowers to $6 \times 10^{-3}$ in GCs and $\sim 10^{-4}$ in YSCs. In the most optimistic case (i.e., when low spins are assumed for $1 \mathrm{~g}$ BHs), $f_{>1 g} \sim 0.5,0.1,0.01$ for NSCs, GCs and YSCs, respectively. In the most pessimistic case (i.e., when high spins are assumed), $f_{>1 g} \sim 0.08,2 \times 10^{-3}$ and $4 \times 10^{-5}$ for NSCs, GCs and YSCs, respectively.

$\mathrm{BH}$ in the pair instability mass gap and IMBHs can form via hierarchical mergers. Their fraction is strongly suppressed at high metallicity. At $Z=0.0002$ and in our fiducial model, the fraction of $\mathrm{BBH}$ mergers with primary $\mathrm{BH}$ mass in the pair instability gap is $f_{\text {PISN }} \sim 7 \times 10^{-3}, 3 \times 10^{-4}$ and $5 \times 10^{-6}$ in NSCs, GCs and YSCs, respectively. In our fiducial model, the fraction of $\mathrm{BBH}$ mergers with primary $\mathrm{BH}$ mass in the $\mathrm{IMBH}$ regime is $f_{\mathrm{IMBH}} \sim 5 \times 10^{-4}$ in NSCs, while we do not find any IMBH mergers in either GCs or YSCs. These fractions are significantly higher in the SMALL_M2 and HIGH_MASS models (Figure 7).

The local BBH merger rates in our models range from $\sim 10$ to $\sim 60 \mathrm{Gpc}^{-3} \mathrm{yr}^{-1}$, but $\mathrm{Ng}$ BBHs in NSCs only account for $10^{-2}-0.2 \mathrm{Gpc}^{-3} \mathrm{yr}^{-1}$ in our models. If we assume that $10 \%$ of all stars form in NSCs, we find a robust upper limit $\sim 7-10 \mathrm{Gpc}^{-3} \mathrm{yr}^{-1}$ for the local merger rate density of $\mathrm{Ng} \mathrm{BBH}$ in NSCs.

We compare our models against LIGO-Virgo data from the second gravitational wave transient catalog (GWTC-2, $[9,10])$ by estimating the mixing fractions of the four considered channels. Even if the mixing fractions are wildly affected by model hyperparameters, our analysis suggests that more than one channel is needed to explain the observed population from GWTC-2. This result confirms that the BBHs observed by the LIGO-Virgo collaboration likely are a combination of several different channels and opens new perspectives for the study of $\mathrm{BBH}$ formation. 
Author Contributions: Conceptualization, M.M., M.A.S. and M.C.A.; methodology, M.M., F.S. and Y.B.; software, M.M., F.S. and Y.B.; writing-original draft preparation, M.M. and A.B.; project administration, M.M.; funding acquisition, M.M., M.C.A. and M.A.S. All authors have read and agreed to the published version of the manuscript.

Funding: M.M., F.S., Y.B. and A.B. acknowledge financial support from the European Research Council for the ERC Consolidator grant DEMOBLACK, under contract no. 770017. M.M. and M.C.A. acknowledges financial support from the Austrian National Science Foundation through FWF standalone grant P31154-N27. M.A.S. acknowledges financial support from the Alexander von Humboldt Foundation for the research program "The evolution of black holes from stellar to galactic scales", the Volkswagen Foundation Trilateral Partnership for project No. I/97778 “Dynamical Mechanisms of Accretion in Galactic Nuclei", and the Deutsche Forschungsgemeinschaft (DFG, German Research Foundation)-Project-ID 138713538-SFB 881 “The Milky Way System”.

Data Availability Statement: The data underlying this article will be shared on reasonable request to the corresponding authors.

Acknowledgments: We thank Eugenio Carretta for useful discussion and we thank the internal referee of the LIGO-Virgo collaboration, Fabio Antonini, for his suggestions, which helped us improve this work.

Conflicts of Interest: The authors declare no conflict of interest. The funders had no role in the design of the study; in the collection, analyses, or interpretation of data; in the writing of the manuscript, or in the decision to publish the results.

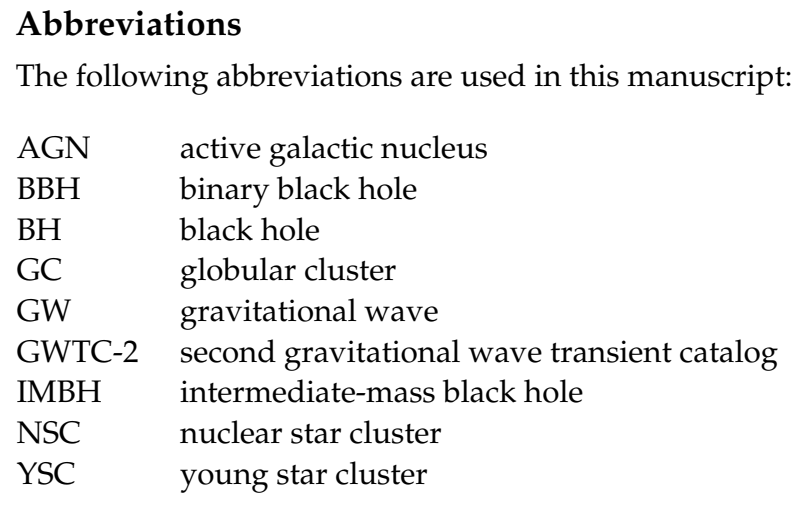

\section{References}

1. Acernese, F.; Agathos, M.; Agatsuma, K.; Aisa, D.; Allemandou, N.; Allocca, A.; Amarni, J.; Astone, P.; Balestri, G.; Ballardin, G.; et al. Advanced Virgo: A second-generation interferometric gravitational wave detector. Class. Quantum Gravity 2015, 32, 024001. [CrossRef]

2. Aasi, J.; Abbott, B.P.; Abbott, R.; Abbott, T.; Abernathy, M.R.; Ackley, K.; Adams, C.; Adams, T.; Addesso, P.; Adhikari, R.X.; et al. Advanced LIGO. Class. Quantum Gravity 2015, 32, 074001.

3. Abbott, B.P.; Abbott, R.; Abbott, T.D.; Abernathy, M.R.; Acernese, F.; Ackley, K.; Adams, C.; Adams, T.; Addesso, P.; Adhikari, R.X.; et al. Observation of Gravitational Waves from a Binary Black Hole Merger. Phys. Rev. Lett. 2016, 116, 061102. [CrossRef] [PubMed]

4. Abbott, B.P.; Abbott, R.; Abbott, T.D.; Abernathy, M.R.; Acernese, F.; Ackley, K.; Adams, C.; Adams, T.; Addesso, P.; Adhikari, R.X.; et al. Astrophysical Implications of the Binary Black-hole Merger GW150914. Astrophys. J. Lett. 2016, 818, L22. [CrossRef]

5. Abbott, B.P.; Abbott, R.; Abbott, T.D.; Abernathy, M.R.; Acernese, F.; Ackley, K.; Adams, C.; Adams, T.; Addesso, P.; Adhikari, R.X.; et al. Binary Black Hole Mergers in the First Advanced LIGO Observing Run. Phys. Rev. X 2016, 6, 041015. [CrossRef]

6. Abbott, B.P.; Abbott, R.; Abbott, T.D.; Acernese, F.; Ackley, K.; Adams, C.; Adams, T.; Addesso, P.; Adhikari, R.X.; Adya, V.B.; et al GW170817: Observation of Gravitational Waves from a Binary Neutron Star Inspiral. Phys. Rev. Lett. 2017, 119, 161101. [CrossRef] [PubMed]

7. Abbott, B.P.; Abbott, R.; Abbott, T.D.; Abraham, S.; Acernese, F.; Ackley, K.; Adams, C.; Adhikari, R.X.; Adya, V.B.; Affeldt, C.; et al. GWTC-1: A Gravitational-Wave Transient Catalog of Compact Binary Mergers Observed by LIGO and Virgo during the First and Second Observing Runs. Phys. Rev. X 2019, 9, 031040. [CrossRef]

8. Abbott, B.P.; Abbott, R.; Abbott, T.D.; Abraham, S.; Acernese, F.; Ackley, K.; Adams, C.; Adhikari, R.X.; Adya, V.B.; Affeldt, C.; et al. Binary Black Hole Population Properties Inferred from the First and Second Observing Runs of Advanced LIGO and Advanced Virgo. Astrophys. J. Lett. 2019, 882, L24. [CrossRef] 
9. Abbott, R.; Abbott, T.D.; Abraham, S.; Acernese, F.; Ackley, K.; Adams, A.; Adams, C.; Adhikari, R.X.; Adya, V.B.; Affeldt, C.; et al. GWTC-2: Compact Binary Coalescences Observed by LIGO and Virgo during the First Half of the Third Observing Run. Phys. Rev. X 2021, 11, 021053. [CrossRef]

10. Abbott, R.; Abbott, T.D.; Abraham, S.; Acernese, F.; Ackley, K.; Adams, A.; Adams, C.; Adhikari, R.X.; Adya, V.B.; Affeldt, C.; et al. Population Properties of Compact Objects from the Second LIGO-Virgo Gravitational-Wave Transient Catalog. Astrophys. J. Lett. 2021, 913, L7. [CrossRef]

11. Abbott, R.; Abbott, T.D.; Acernese, F.; Ackley, K.; Adams, C.; Adhikari, N.; Adhikari, R.X.; Adya, V.B.; Affeldt, C.; Agarwal, D.; et al. GWTC-2.1: Deep Extended Catalog of Compact Binary Coalescences Observed by LIGO and Virgo During the First Half of the Third Observing Run. arXiv 2021, arXiv:2108.01045.

12. Carr, B.J.; Hawking, S.W. Black holes in the early Universe. Mon. Not. R. Astron. Soc. 1974, 168, 399-416. [CrossRef]

13. Carr, B.; Kühnel, F.; Sandstad, M. Primordial black holes as dark matter. Phys. Rev. D 2016, 94, 083504. [CrossRef]

14. Bird, S.; Cholis, I.; Muñoz, J.B.; Ali-Haïmoud, Y.; Kamionkowski, M.; Kovetz, E.D.; Raccanelli, A.; Riess, A.G. Did LIGO Detect Dark Matter? Phys. Rev. Lett. 2016, 116, 201301. [CrossRef]

15. Tutukov, A.; Yungelson, L. Evolution of massive close binaries. Nauchnye Informatsii 1973, 27, 70.

16. Bethe, H.A.; Brown, G.E. Evolution of Binary Compact Objects That Merge. Astrophys. J. 1998, 506, 780-789. [CrossRef]

17. Portegies Zwart, S.F.; Yungelson, L.R. Formation and evolution of binary neutron stars. Astron. Astrophys. 1998, 332, $173-188$.

18. Belczynski, K.; Kalogera, V.; Bulik, T. A Comprehensive Study of Binary Compact Objects as Gravitational Wave Sources: Evolutionary Channels, Rates, and Physical Properties. Astrophys. J. 2002, 572, 407-431. [CrossRef]

19. Voss, R.; Tauris, T.M. Galactic distribution of merging neutron stars and black holes-prospects for short gamma-ray burst progenitors and LIGO/VIRGO. Mon. Not. R. Astron. Soc. 2003, 342, 1169-1184. [CrossRef]

20. Podsiadlowski, P.; Langer, N.; Poelarends, A.J.T.; Rappaport, S.; Heger, A.; Pfahl, E. The Effects of Binary Evolution on the Dynamics of Core Collapse and Neutron Star Kicks. Astrophys. J. 2004, 612, 1044-1051. [CrossRef]

21. Belczynski, K.; Kalogera, V.; Rasio, F.A.; Taam, R.E.; Zezas, A.; Bulik, T.; Maccarone, T.J.; Ivanova, N. Compact Object Modeling with the StarTrack Population Synthesis Code. Astrophys. J. Suppl. 2008, 174, 223-260. [CrossRef]

22. Dominik, M.; Belczynski, K.; Fryer, C.; Holz, D.E.; Berti, E.; Bulik, T.; Mandel, I.; O'Shaughnessy, R. Double Compact Objects. I. The Significance of the Common Envelope on Merger Rates. Astrophys. J. 2012, 759, 52. [CrossRef]

23. Dominik, M.; Belczynski, K.; Fryer, C.; Holz, D.E.; Berti, E.; Bulik, T.; Mandel, I.; O'Shaughnessy, R. Double Compact Objects. II. Cosmological Merger Rates. Astrophys. J. 2013, 779, 72. [CrossRef]

24. Mennekens, N.; Vanbeveren, D. Massive double compact object mergers: Gravitational wave sources and r-process element production sites. Astron. Astrophys. 2014, 564, A134. [CrossRef]

25. Belczynski, K.; Holz, D.E.; Bulik, T.; O'Shaughnessy, R. The first gravitational-wave source from the isolated evolution of two stars in the 40-100 solar mass range. Mon. Not. R. Astron. Soc. 2016, 534, 512-515. [CrossRef] [PubMed]

26. Eldridge, J.J.; Stanway, E.R. BPASS predictions for binary black hole mergers. Mon. Not. R. Astron. Soc. 2016, 462, 3302-3313. [CrossRef]

27. Stevenson, S.; Berry, C.P.L.; Mandel, I. Hierarchical analysis of gravitational-wave measurements of binary black hole spin-orbit misalignments. arXiv 2017, arXiv:1703.06873.

28. Mapelli, M.; Giacobbo, N.; Ripamonti, E.; Spera, M. The cosmic merger rate of stellar black hole binaries from the Illustris simulation. Mon. Not. R. Astron. Soc. 2017, 472, 2422-2435. [CrossRef]

29. Mapelli, M.; Giacobbo, N. The cosmic merger rate of neutron stars and black holes. Mon. Not. R. Astron. Soc. 2018, 479, 4391-4398. [CrossRef]

30. Giacobbo, N.; Mapelli, M.; Spera, M. Merging black hole binaries: The effects of progenitor's metallicity, mass-loss rate and Eddington factor. Mon. Not. R. Astron. Soc. 2018, 474, 2959-2974. [CrossRef]

31. Giacobbo, N.; Mapelli, M. The progenitors of compact-object binaries: Impact of metallicity, common envelope and natal kicks. Mon. Not. R. Astron. Soc. 2018, 480, 2011-2030. [CrossRef]

32. Klencki, J.; Moe, M.; Gladysz, W.; Chruslinska, M.; Holz, D.E.; Belczynski, K. Impact of inter-correlated initial binary parameters on double black hole and neutron star mergers. Astron. Astrophys. 2018, 619, A77. [CrossRef]

33. Kruckow, M.U.; Tauris, T.M.; Langer, N.; Kramer, M.; Izzard, R.G. Progenitors of gravitational wave mergers: Binary evolution with the stellar grid-based code COMBINE. Mon. Not. R. Astron. Soc. 2018, 481, 1908-1949. [CrossRef]

34. Spera, M.; Mapelli, M.; Giacobbo, N.; Trani, A.A.; Bressan, A.; Costa, G. Merging black hole binaries with the SEVN code. Mon. Not. R. Astron. Soc. 2019, 485, 889-907. [CrossRef]

35. Mapelli, M.; Giacobbo, N.; Santoliquido, F.; Artale, M.C. The properties of merging black holes and neutron stars across cosmic time. Mon. Not. R. Astron. Soc. 2019, 487, 2-13. [CrossRef]

36. Neijssel, C.J.; Vigna-Gómez, A.; Stevenson, S.; Barrett, J.W.; Gaebel, S.M.; Broekgaarden, F.S.; de Mink, S.E.; Szécsi, D.; Vinciguerra, S.; Mandel, I. The effect of the metallicity-specific star formation history on double compact object mergers. Mon. Not. R. Astron. Soc. 2019, 490, 3740-3759. [CrossRef]

37. Eldridge, J.J.; Stanway, E.R.; Tang, P.N. A consistent estimate for gravitational wave and electromagnetic transient rates. Mon. Not. R. Astron. Soc. 2019, 482, 870-880. [CrossRef]

38. Tang, P.N.; Eldridge, J.J.; Stanway, E.R.; Bray, J.C. Dependence of Gravitational Wave Transient Rates on Cosmic Star Formation and Metallicity Evolution History. arXiv 2019, arXiv:1912.04474. 
39. Marchant, P.; Langer, N.; Podsiadlowski, P.; Tauris, T.M.; Moriya, T.J. A new route towards merging massive black holes. Astron. Astrophys. 2016, 588, A50. [CrossRef]

40. de Mink, S.E.; Mandel, I. The chemically homogeneous evolutionary channel for binary black hole mergers: Rates and properties of gravitational-wave events detectable by advanced LIGO. Mon. Not. R. Astron. Soc. 2016, 460, 3545-3553. [CrossRef]

41. Mandel, I.; de Mink, S.E. Merging binary black holes formed through chemically homogeneous evolution in short-period stellar binaries. Mon. Not. R. Astron. Soc. 2016, 458, 2634-2647. [CrossRef]

42. du Buisson, L.; Marchant, P.; Podsiadlowski, P.; Kobayashi, C.; Abdalla, F.B.; Taylor, P.; Mandel, I.; de Mink, S.E.; Moriya, T.J.; Langer, N. Cosmic Rates of Black Hole Mergers and Pair-Instability Supernovae from Chemically Homogeneous Binary Evolution. arXiv 2020, arXiv:2002.11630.

43. Antonini, F.; Chatterjee, S.; Rodriguez, C.L.; Morscher, M.; Pattabiraman, B.; Kalogera, V.; Rasio, F.A. Black Hole Mergers and Blue Stragglers from Hierarchical Triples Formed in Globular Clusters. Astrophys. J. 2016, 816, 65. [CrossRef]

44. Antonini, F.; Toonen, S.; Hamers, A.S. Binary Black Hole Mergers from Field Triples: Properties, Rates, and the Impact of Stellar Evolution. Astrophys. J. 2017, 841, 77. [CrossRef]

45. Arca-Sedda, M.; Li, G.; Kocsis, B. Ordering the chaos: Stellar black hole mergers from non-hierarchical triples. arXiv 2018, arXiv:1805.06458.

46. Fragione, G.; Loeb, A. Black hole-neutron star mergers from triples. Mon. Not. R. Astron. Soc. 2019, 486, 4443-4450. [CrossRef]

47. Fragione, G.; Silk, J. Repeated mergers and ejection of black holes within nuclear star clusters. arXiv 2020, arXiv:2006.01867.

48. Banerjee, S.; Baumgardt, H.; Kroupa, P. Stellar-mass black holes in star clusters: Implications for gravitational wave radiation. Mon. Not. R. Astron. Soc. 2010, 402, 371-380. [CrossRef]

49. Ziosi, B.M.; Mapelli, M.; Branchesi, M.; Tormen, G. Dynamics of stellar black holes in young star clusters with different metallicitiesII. Black hole-black hole binaries. Mon. Not. R. Astron. Soc. 2014, 441, 3703-3717. [CrossRef]

50. Mapelli, M. Massive black hole binaries from runaway collisions: The impact of metallicity. Mon. Not. R. Astron. Soc. 2016, 459, 3432-3446. [CrossRef]

51. Askar, A.; Szkudlarek, M.; Gondek-Rosińska, D.; Giersz, M.; Bulik, T. MOCCA-SURVEY DatabaseI. Coalescing binary black holes originating from globular clusters. Mon. Not. R. Astron. Soc. 2017, 464, L36-L40. [CrossRef]

52. Banerjee, S. Stellar-mass black holes in young massive and open stellar clusters and their role in gravitational-wave generation. Mon. Not. R. Astron. Soc. 2017, 467, 524-539. [CrossRef]

53. Banerjee, S. Stellar-mass black holes in young massive and open stellar clusters and their role in gravitational-wave generation II. Mon. Not. R. Astron. Soc. 2018, 473, 909-926. [CrossRef]

54. Banerjee, S. Stellar-mass black holes in young massive and open stellar clusters and their role in gravitational-wave generation IV: Updated stellar-evolutionary and black hole spin models and comparisons with the LIGO-Virgo O1/O2 merger-event data. arXiv 2020, arXiv:2004.07382.

55. Di Carlo, U.N.; Giacobbo, N.; Mapelli, M.; Pasquato, M.; Spera, M.; Wang, L.; Haardt, F. Merging black holes in young star clusters. Mon. Not. R. Astron. Soc. 2019, 487, 2947-2960. [CrossRef]

56. Di Carlo, U.N.; Mapelli, M.; Bouffanais, Y.; Giacobbo, N.; Bressan, S.; Spera, M.; Haardt, F. Binary black holes in the pair-instability mass gap. Mon. Not. R. Astron. Soc. 2020, 497, 1043-1049. [CrossRef]

57. Kumamoto, J.; Fujii, M.S.; Tanikawa, A. Gravitational-wave emission from binary black holes formed in open clusters. Mon. Not. R. Astron. Soc. 2019, 486, 3942-3950. [CrossRef]

58. Kumamoto, J.; Fujii, M.S.; Tanikawa, A. Merger rate density of binary black holes formed in open clusters. arXiv 2020, arXiv:2001.10690.

59. Portegies Zwart, S.F.; McMillan, S.L.W. Black Hole Mergers in the Universe. Astrophys. J. Lett. 2000, 528, L17-L20. [CrossRef]

60. Downing, J.M.B.; Benacquista, M.J.; Giersz, M.; Spurzem, R. Compact binaries in star clustersI. Black hole binaries inside globular clusters. Mon. Not. R. Astron. Soc. 2010, 407, 1946-1962. [CrossRef]

61. Rodriguez, C.L.; Morscher, M.; Pattabiraman, B.; Chatterjee, S.; Haster, C.J.; Rasio, F.A. Binary Black Hole Mergers from Globular Clusters: Implications for Advanced LIGO. Phys. Rev. Lett. 2015, 115, 051101. [CrossRef] [PubMed]

62. Rodriguez, C.L.; Chatterjee, S.; Rasio, F.A. Binary black hole mergers from globular clusters: Masses, merger rates, and the impact of stellar evolution. Phys. Rev. D 2016, 93, 084029. [CrossRef]

63. Rodriguez, C.L.; Amaro-Seoane, P.; Chatterjee, S.; Kremer, K.; Rasio, F.A.; Samsing, J.; Ye, C.S.; Zevin, M. Post-Newtonian dynamics in dense star clusters: Formation, masses, and merger rates of highly-eccentric black hole binaries. Phys. Rev. D 2018, 98, 123005. [CrossRef]

64. Samsing, J.; MacLeod, M.; Ramirez-Ruiz, E. The Formation of Eccentric Compact Binary Inspirals and the Role of Gravitational Wave Emission in Binary-Single Stellar Encounters. Astrophys. J. 2014, 784, 71. [CrossRef]

65. Samsing, J. Eccentric black hole mergers forming in globular clusters. Phys. Rev. D 2018, 97, 103014, [CrossRef]

66. Fragione, G.; Kocsis, B. Black Hole Mergers from an Evolving Population of Globular Clusters. Phys. Rev. Lett. 2018, 121, 161103. [CrossRef] [PubMed]

67. Zevin, M.; Samsing, J.; Rodriguez, C.; Haster, C.J.; Ramirez-Ruiz, E. Eccentric Black Hole Mergers in Dense Star Clusters: The Role of Binary-Binary Encounters. Astrophys. J. 2019, 871, 91. [CrossRef] 
68. Antonini, F.; Gieles, M. Population synthesis of black hole binary mergers from star clusters. Mon. Not. R. Astron. Soc. 2020, 492, 2936-2954. [CrossRef]

69. O'Leary, R.M.; Kocsis, B.; Loeb, A. Gravitational waves from scattering of stellar-mass black holes in galactic nuclei. Mon. Not. R. Astron. Soc. 2009, 395, 2127-2146. [CrossRef]

70. Miller, M.C.; Lauburg, V.M. Mergers of Stellar-Mass Black Holes in Nuclear Star Clusters. Astrophys. J. 2009, 692, 917-923. [CrossRef]

71. Antonini, F.; Rasio, F.A. Merging Black Hole Binaries in Galactic Nuclei: Implications for Advanced-LIGO Detections. Astrophys. J. 2016, 831, 187. [CrossRef]

72. Petrovich, C.; Antonini, F. Greatly Enhanced Merger Rates of Compact-object Binaries in Non-spherical Nuclear Star Clusters Astrophys. J. 2017, 846, 146. [CrossRef]

73. Rasskazov, A.; Kocsis, B. The Rate of Stellar Mass Black Hole Scattering in Galactic Nuclei. Astrophys. J. 2019, 881, 20. [CrossRef]

74. Arca-Sedda, M.; Gualandris, A. Gravitational wave sources from inspiralling globular clusters in the Galactic Centre and similar environments. Mon. Not. R. Astron. Soc. 2018, 477, 4423-4442. [CrossRef]

75. Arca Sedda, M.; Benacquista, M. Using final black hole spins and masses to infer the formation history of the observed population of gravitational wave sources. Mon. Not. R. Astron. Soc. 2019, 482, 2991-3010. [CrossRef]

76. Arca Sedda, M.; Mapelli, M.; Spera, M.; Benacquista, M.; Giacobbo, N. Fingerprints of Binary Black Hole Formation Channels Encoded in the Mass and Spin of Merger Remnants. Astrophys. J. 2020, 894, 133. [CrossRef]

77. Arca Sedda, M. Birth, Life, and Death of Black Hole Binaries around Supermassive Black Holes: Dynamical Evolution of Gravitational Wave Sources. Astrophys. J. 2020, 891, 47. [CrossRef]

78. McKernan, B.; Ford, K.E.S.; Lyra, W.; Perets, H.B. Intermediate mass black holes in AGN discsI. Production and growth. Mon. Not. R. Astron. Soc. 2012, 425, 460-469. [CrossRef]

79. McKernan, B.; Ford, K.E.S.; Bellovary, J.; Leigh, N.W.C.; Haiman, Z.; Kocsis, B.; Lyra, W.; Mac Low, M.M.; Metzger, B.; O’Dowd, M.; et al. Constraining Stellar-mass Black Hole Mergers in AGN Disks Detectable with LIGO. Astrophys. J. 2018, 866, 66. [CrossRef]

80. Bartos, I.; Kocsis, B.; Haiman, Z.; Márka, S. Rapid and Bright Stellar-mass Binary Black Hole Mergers in Active Galactic Nuclei. Astrophys. J. 2017, 835, 165. [CrossRef]

81. Stone, N.C.; Metzger, B.D.; Haiman, Z. Assisted inspirals of stellar mass black holes embedded in AGN discs: Solving the 'final au problem'. Mon. Not. R. Astron. Soc. 2017, 464, 946-954. [CrossRef]

82. Yang, Y.; Bartos, I.; Haiman, Z.; Kocsis, B.; Márka, Z.; Stone, N.C.; Márka, S. AGN Disks Harden the Mass Distribution of Stellar-mass Binary Black Hole Mergers. Astrophys. J. 2019, 876, 122. [CrossRef]

83. Tagawa, H.; Haiman, Z.; Kocsis, B. Formation and Evolution of Compact Object Binaries in AGN Disks. arXiv 2019, arXiv:1912.08218.

84. Miller, M.C.; Hamilton, D.P. Production of intermediate-mass black holes in globular clusters. Mon. Not. R. Astron. Soc. 2002, 330, 232-240. [CrossRef]

85. Fishbach, M.; Holz, D.E.; Farr, B. Are LIGO's Black Holes Made from Smaller Black Holes? Astrophys. J. Lett. 2017, 840, L24. [CrossRef]

86. Gerosa, D.; Berti, E. Are merging black holes born from stellar collapse or previous mergers? Phys. Rev. D 2017, 95, 124046. [CrossRef]

87. Doctor, Z.; Wysocki, D.; O’Shaughnessy, R.; Holz, D.E.; Farr, B. Black Hole Coagulation: Modeling Hierarchical Mergers in Black Hole Populations. Astrophys. J. 2020, 893, 35. [CrossRef]

88. Kimball, C.; Talbot, C.; Berry, C.P.L.; Carney, M.; Zevin, M.; Thrane, E.; Kalogera, V. Black hole genealogy: Identifying hierarchical mergers with gravitational waves. arXiv 2020, arXiv:2005.00023.

89. Hills, J.G.; Fullerton, L.W. Computer simulations of close encounters between single stars and hard binaries. Astron. J. 1980, 85, 1281-1291. [CrossRef]

90. Fitchett, M.J. The influence of gravitational wave momentum losses on the centre of mass motion of a Newtonian binay system. Mon. Not. R. Astron. Soc. 1983, 203, 1049-1062. [CrossRef]

91. Favata, M.; Hughes, S.A.; Holz, D.E. How Black Holes Get Their Kicks: Gravitational Radiation Recoil Revisited. Astrophys. J. Lett. 2004, 607, L5-L8. [CrossRef]

92. Campanelli, M.; Lousto, C.; Zlochower, Y.; Merritt, D. Large Merger Recoils and Spin Flips from Generic Black Hole Binaries. Astrophys. J. Lett. 2007, 659, L5-L8. [CrossRef]

93. Lousto, C.O.; Zlochower, Y. Hangup Kicks: Still Larger Recoils by Partial Spin-Orbit Alignment of Black-Hole Binaries. Phys. Rev. Lett. 2011, 107, 231102. [CrossRef] [PubMed]

94. Holley-Bockelmann, K.; Gültekin, K.; Shoemaker, D.; Yunes, N. Gravitational Wave Recoil and the Retention of Intermediate-Mass Black Holes. Astrophys. J. 2008, 686, 829-837. [CrossRef]

95. Moody, K.; Sigurdsson, S. Modeling the Retention Probability of Black Holes in Globular Clusters: Kicks and Rates. Astrophys. J. 2009, 690, 1370-1377. [CrossRef]

96. Fragione, G.; Ginsburg, I.; Kocsis, B. Gravitational Waves and Intermediate-mass Black Hole Retention in Globular Clusters. Astrophys. J. 2018, 856, 92. [CrossRef] 
97. Gerosa, D.; Berti, E. Escape speed of stellar clusters from multiple-generation black-hole mergers in the upper mass gap. arXiv 2019, arXiv:1906.05295.

98. Lousto, C.O.; Zlochower, Y.; Dotti, M.; Volonteri, M. Gravitational recoil from accretion-aligned black-hole binaries. Phys. Rev. D 2012, 85, 084015. [CrossRef]

99. Maggiore, M. Gravitational Waves: Volume 2: Astrophysics and Cosmology; Gravitational Waves; Oxford University Press: Oxford, UK, 2018.

100. Rodriguez, C.L.; Zevin, M.; Amaro-Seoane, P.; Chatterjee, S.; Kremer, K.; Rasio, F.A.; Ye, C.S. Black Holes: The Next GenerationRepeated Mergers in Dense Star Clusters and their Gravitational-Wave Properties. arXiv 2019, arXiv:1906.10260.

101. Arca-Sedda, M.; Capuzzo-Dolcetta, R. The MEGaN project II. Gravitational waves from intermediate-mass and binary black holes around a supermassive black hole. Mon. Not. R. Astron. Soc. 2019, 483, 152-171. [CrossRef]

102. Antonini, F.; Gieles, M.; Gualandris, A. Black hole growth through hierarchical black hole mergers in dense star clusters: Implications for gravitational wave detections. Mon. Not. R. Astron. Soc. 2019, 486, 5008-5021. [CrossRef]

103. Belczynski, K.; Heger, A.; Gladysz, W.; Ruiter, A.J.; Woosley, S.; Wiktorowicz, G.; Chen, H.Y.; Bulik, T.; O'Shaughnessy, R.; Holz, D.E.; et al. The effect of pair-instability mass loss on black-hole mergers. Astron. Astrophys. 2016, 594, A97. [CrossRef]

104. Woosley, S.E. Pulsational Pair-instability Supernovae. Astrophys. J. 2017, 836, 244. [CrossRef]

105. Spera, M.; Mapelli, M. Very massive stars, pair-instability supernovae and intermediate-mass black holes with the sevn code. Mon. Not. R. Astron. Soc. 2017, 470, 4739-4749. [CrossRef]

106. Farmer, R.; Renzo, M.; de Mink, S.E.; Marchant, P.; Justham, S. Mind the Gap: The Location of the Lower Edge of the Pair-instability Supernova Black Hole Mass Gap. Astrophys. J. 2019, 887, 53. [CrossRef]

107. Mapelli, M.; Spera, M.; Montanari, E.; Limongi, M.; Chieffi, A.; Giacobbo, N.; Bressan, A.; Bouffanais, Y. Impact of the Rotation and Compactness of Progenitors on the Mass of Black Holes. Astrophys. J. 2020, 888, 76. [CrossRef]

108. Renzo, M.; Farmer, R.J.; Justham, S.; de Mink, S.E.; Götberg, Y.; Marchant, P. Sensitivity of the lower-edge of the pair instability black hole mass gap to the treatment of time dependent convection. Mon. Not. R. Astron. Soc. 2020, 493, 4333-4341. [CrossRef]

109. Abbott, R.; Abbott, T.D.; Abraham, S.; Acernese, F.; Ackley, K.; Adams, C.; Adhikari, R.X.; Adya, V.B.; Affeldt, C.; Agathos, M.; et al. GW190521: A Binary Black Hole Merger with a Total Mass of $150 \mathrm{M}_{\odot}$. Phys. Rev. Lett. 2020, 125, 101102. [CrossRef] [PubMed]

110. Abbott, R.; Abbott, T.D.; Abraham, S.; Acernese, F.; Ackley, K.; Adams, C.; Adhikari, R.X.; Adya, V.B.; Affeldt, C.; Agathos, M.; et al. Properties and Astrophysical Implications of the $150 \mathrm{M}_{\odot}$ Binary Black Hole Merger GW190521. Astrophys. J. Lett. 2020, 900, L13. [CrossRef]

111. Kimball, C.; Talbot, C.; Berry, C.P.L.; Zevin, M.; Thrane, E.; Kalogera, V.; Buscicchio, R.; Carney, M.; Dent, T.; Middleton, H.; et al. Evidence for hierarchical black hole mergers in the second LIGO-Virgo gravitational-wave catalog. arXiv 2020, arXiv:2011.05332.

112. Fragione, G.; Loeb, A.; Rasio, F.A. On the Origin of GW190521-like Events from Repeated Black Hole Mergers in Star Clusters. Astrophys. J. Lett. 2020, 902, L26. [CrossRef]

113. Rizzuto, F.P.; Naab, T.; Spurzem, R.; Giersz, M.; Ostriker, J.P.; Stone, N.C.; Wang, L.; Berczik, P.; Rampp, M. Intermediate mass black hole formation in compact young massive star clusters. Mon. Not. R. Astron. Soc. 2021, 501, 5257-5273. [CrossRef]

114. Liu, B.; Lai, D. Hierarchical Black-Hole Mergers in Multiple Systems: Constrain the Formation of GW190412, GW190814 and GW190521-like events. Mon. Not. R. Astron. Soc. 2021, 502, 2049-2064. [CrossRef]

115. Mapelli, M.; Dall'Amico, M.; Bouffanais, Y.; Giacobbo, N.; Arca Sedda, M.; Artale, M.C.; Ballone, A.; Di Carlo, U.N.; Iorio, G.; Santoliquido, F.; et al. Hierarchical black hole mergers in young, globular and nuclear star clusters: The effect of metallicity, spin and cluster properties. Mon. Not. R. Astron. Soc. 2021, 505, 339-358. [CrossRef]

116. Dall'Amico, M.; Mapelli, M.; Niccolò Di Carlo, U.; Bouffanais, Y.; Rastello, S.; Santoliquido, F.; Ballone, A.; Arca Sedda, M. GW190521 formation via three-body encounters in young massive star clusters. arXiv 2021, arXiv:2105.12757.

117. Kremer, K.; Spera, M.; Becker, D.; Chatterjee, S.; Di Carlo, U.N.; Fragione, G.; Rodriguez, C.L.; Ye, C.S.; Rasio, F.A. Populating the Upper Black Hole Mass Gap through Stellar Collisions in Young Star Clusters. Astrophys. J. 2020, 903, 45. [CrossRef]

118. Roupas, Z.; Kazanas, D. Generation of massive stellar black holes by rapid gas accretion in primordial dense clusters. Astron. Astrophys. 2019, 632, L8. [CrossRef]

119. Rice, J.R.; Zhang, B. Growth of Stellar-mass Black Holes in Dense Molecular Clouds and GW190521. Astrophys. J. 2021, 908, 59. [CrossRef]

120. Safarzadeh, M.; Haiman, Z. Formation of GW190521 via Gas Accretion onto Population III Stellar Black Hole Remnants Born in High-redshift Minihalos. Astrophys. J. Lett. 2020, 903, L21. [CrossRef]

121. Palmese, A.; Conselice, C.J. GW190521 from the Merger of Ultra-Dwarf Galaxies. arXiv 2020, arXiv:2009.10688.

122. Belczynski, K. The Most Ordinary Formation of the Most Unusual Double Black Hole Merger. Astrophys. J. Lett. 2020, 905, L15. [CrossRef]

123. Farrell, E.; Groh, J.H.; Hirschi, R.; Murphy, L.; Kaiser, E.; Ekström, S.; Georgy, C.; Meynet, G. Is GW190521 the merger of black holes from the first stellar generations? Mon. Not. R. Astron. Soc. 2021, 502, L40-L44. [CrossRef]

124. Tanikawa, A.; Kinugawa, T.; Yoshida, T.; Hijikawa, K.; Umeda, H. Population III Binary Black Holes: Effects of Convective Overshooting on Formation of GW190521. arXiv 2020, arXiv:2010.07616. 
125. Costa, G.; Bressan, A.; Mapelli, M.; Marigo, P.; Iorio, G.; Spera, M. Formation of GW190521 from stellar evolution: The impact of the hydrogen-rich envelope, dredge-up, and ${ }^{12} \mathrm{C}(\alpha, \gamma){ }^{16} \mathrm{O}$ rate on the pair-instability black hole mass gap. Mon. Not. R. Astron. Soc. 2021, 501, 4514-4533. [CrossRef]

126. De Luca, V.; Desjacques, V.; Franciolini, G.; Pani, P.; Riotto, A. GW190521 Mass Gap Event and the Primordial Black Hole Scenario. Phys. Rev. Lett. 2021, 126, 051101. [CrossRef] [PubMed]

127. Portegies Zwart, S.F.; McMillan, S.L.W.; Gieles, M. Young Massive Star Clusters. Annu. Rev. Astron. Astrophys. 2010, $48,431-493$. [CrossRef]

128. Hurley, J.R.; Tout, C.A.; Pols, O.R. Evolution of binary stars and the effect of tides on binary populations. Mon. Not. R. Astron. Soc. 2002, 329, 897-928. [CrossRef]

129. Smith, N. Mass Loss: Its Effect on the Evolution and Fate of High-Mass Stars. Annu. Rev. Astron. Astrophys. 2014, 52, 487-528. [CrossRef]

130. Chen, Y.; Bressan, A.; Girardi, L.; Marigo, P.; Kong, X.; Lanza, A. PARSEC evolutionary tracks of massive stars up to $350 \mathrm{M}_{\odot}$ at metallicities $0.0001 \leq \mathrm{Z} \leq 0.04$. Mon. Not. R. Astron. Soc. 2015, 452, 1068-1080. [CrossRef]

131. Gräfener, G.; Hamann, W.R. Mass loss from late-type WN stars and its Z-dependence. Very massive stars approaching the Eddington limit. Astron. Astrophys. 2008, 482, 945-960. [CrossRef]

132. Vink, J.S.; de Koter, A.; Lamers, H.J.G.L.M. Mass-loss predictions for O and B stars as a function of metallicity. Astron. Astrophys. 2001, 369, 574-588. [CrossRef]

133. Vink, J.S.; Muijres, L.E.; Anthonisse, B.; de Koter, A.; Gräfener, G.; Langer, N. Wind modelling of very massive stars up to 300 solar masses. Astron. Astrophys. 2011, 531, A132. [CrossRef]

134. Belczynski, K.; Bulik, T.; Fryer, C.L.; Ruiter, A.; Valsecchi, F.; Vink, J.S.; Hurley, J.R. On the Maximum Mass of Stellar Black Holes. Astrophys. J. 2010, 714, 1217-1226. [CrossRef]

135. Hurley, J.R.; Pols, O.R.; Tout, C.A. Comprehensive analytic formulae for stellar evolution as a function of mass and metallicity. Mon. Not. R. Astron. Soc. 2000, 315, 543-569. [CrossRef]

136. Fryer, C.L.; Belczynski, K.; Wiktorowicz, G.; Dominik, M.; Kalogera, V.; Holz, D.E. Compact Remnant Mass Function: Dependence on the Explosion Mechanism and Metallicity. Astrophys. J. 2012, 749, 91. [CrossRef]

137. Timmes, F.X.; Woosley, S.E.; Weaver, T.A. The Neutron Star and Black Hole Initial Mass Function. Astrophys. J. 1996, $457,834$. [CrossRef]

138. Zevin, M.; Spera, M.; Berry, C.P.L.; Kalogera, V. Exploring the Lower Mass Gap and Unequal Mass Regime in Compact Binary Evolution. arXiv 2020, arXiv:2006.14573.

139. Giacobbo, N.; Mapelli, M. The impact of electron-capture supernovae on merging double neutron stars. Mon. Not. R. Astron. Soc. 2019, 482, 2234-2243. [CrossRef]

140. Giacobbo, N.; Mapelli, M. Revising Natal Kick Prescriptions in Population Synthesis Simulations. Astrophys. J. 2020, 891, 141. [CrossRef]

141. Webbink, R.F. Double white dwarfs as progenitors of R Coronae Borealis stars and Type I supernovae. Astrophys. J. 1984, 277, 355-360. [CrossRef]

142. Ivanova, N.; Justham, S.; Chen, X.; De Marco, O.; Fryer, C.L.; Gaburov, E.; Ge, H.; Glebbeek, E.; Han, Z.; Li, X.D.; et al. Common envelope evolution: Where we stand and how we can move forward. Astron. Astrophys. Rev. 2013, 21, 59. [CrossRef]

143. Fragos, T.; Andrews, J.J.; Ramirez-Ruiz, E.; Meynet, G.; Kalogera, V.; Taam, R.E.; Zezas, A. The Complete Evolution of a Neutron-star Binary through a Common Envelope Phase Using 1D Hydrodynamic Simulations. Astrophys. J. Lett. 2019, 883, L45. [CrossRef]

144. Gratton, R.; Sneden, C.; Carretta, E. Abundance Variations Within Globular Clusters. Annu. Rev. Astron. Astrophys. 2004, 42, 385-440. [CrossRef]

145. Fiorentino, G.; Contreras Ramos, R.; Clementini, G.; Marconi, M.; Musella, I.; Aloisi, A.; Annibali, F.; Saha, A.; Tosi, M.; van der Marel, R.P. Multi-Epoch Hubble Space Telescope Observations of IZw18: Characterization of Variable Stars at Ultra-Low Metallicities. Astrophys. J. 2010, 711, 808-817. [CrossRef]

146. Peters, P.C. Gravitational Radiation and the Motion of Two Point Masses. Phys. Rev. 1964, 136, 1224-1232. [CrossRef]

147. Fuller, J.; Ma, L. Most Black Holes Are Born Very Slowly Rotating. Astrophys. J. Lett. 2019, 881, L1, [CrossRef]

148. Belczynski, K.; Klencki, J.; Fields, C.E.; Olejak, A.; Berti, E.; Meynet, G.; Fryer, C.L.; Holz, D.E.; O'Shaughnessy, R.; Brown, D.A.; et al. Evolutionary roads leading to low effective spins, high black hole masses, and O1/O2 rates for LIGO/Virgo binary black holes. Astron. Astrophys. 2020, 636, A104. [CrossRef]

149. Qin, Y.; Fragos, T.; Meynet, G.; Andrews, J.; Sørensen, M.; Song, H.F. The spin of the second-born black hole in coalescing binary black holes. Astron. Astrophys. 2018, 616, A28. [CrossRef]

150. Qin, Y.; Marchant, P.; Fragos, T.; Meynet, G.; Kalogera, V. On the Origin of Black Hole Spin in High-mass X-Ray Binaries. Astrophys. J. Lett. 2019, 870, L18. [CrossRef]

151. Kroupa, P. On the variation of the initial mass function. Mon. Not. R. Astron. Soc. 2001, 322, 231-246. [CrossRef]

152. Sana, H.; de Mink, S.E.; de Koter, A.; Langer, N.; Evans, C.J.; Gieles, M.; Gosset, E.; Izzard, R.G.; Le Bouquin, J.B.; Schneider, F.R.N. Binary Interaction Dominates the Evolution of Massive Stars. Science 2012, 337, 444. [CrossRef]

153. Lousto, C.O.; Zlochower, Y. Modeling gravitational recoil from precessing highly spinning unequal-mass black-hole binaries. Phys. Rev. D 2009, 79, 064018. [CrossRef] 
154. Lousto, C.O.; Zlochower, Y. Further insight into gravitational recoil. Phys. Rev. D 2008, 77, 044028. [CrossRef]

155. Kidder, L.E. Coalescing binary systems of compact objects to (post) ${ }^{5 / 2}$-Newtonian order. V. Spin effects. Phys. Rev. D 1995, 52, 821-847. [CrossRef] [PubMed]

156. Harris, W.E.; Harris, G.L.H.; Alessi, M. A Catalog of Globular Cluster Systems: What Determines the Size of a Galaxy's Globular Cluster Population? Astrophys. J. 2013, 772, 82. [CrossRef]

157. Jiménez-Forteza, X.; Keitel, D.; Husa, S.; Hannam, M.; Khan, S.; Pürrer, M. Hierarchical data-driven approach to fitting numerical relativity data for nonprecessing binary black holes with an application to final spin and radiated energy. Phys. Rev. D 2017, 95, 064024. [CrossRef]

158. Rezzolla, L.; Barausse, E.; Dorband, E.N.; Pollney, D.; Reisswig, C.; Seiler, J.; Husa, S. Final spin from the coalescence of two black holes. Phys. Rev. D 2008, 78, 044002. [CrossRef]

159. Hofmann, F.; Barausse, E.; Rezzolla, L. The Final Spin from Binary Black Holes in Quasi-circular Orbits. Astrophys. J. Lett. 2016, 825, L19. [CrossRef]

160. Piran, T. The Implications of the Compton (GRO) Observations for Cosmological Gamma-Ray Bursts. Astrophys. J. Lett. 1992, 389, L45. [CrossRef]

161. Totani, T.; Morokuma, T.; Oda, T.; Doi, M.; Yasuda, N. Delay Time Distribution Measurement of Type Ia Supernovae by the Subaru/XMM-Newton Deep Survey and Implications for the Progenitor. Publ. Astron. Soc. Jpn. 2008, 60, 1327. [CrossRef]

162. Wang, F.Y.; Wang, Y.Y.; Yang, Y.P.; Yu, Y.W.; Zuo, Z.Y.; Dai, Z.G. Fast Radio Bursts from Activity of Neutron Stars Newborn in BNS Mergers: Offset, Birth Rate, and Observational Properties. Astrophys. J. 2020, 891, 72. [CrossRef]

163. Chandrasekhar, S. Stochastic Problems in Physics and Astronomy. Rev. Mod. Phys. 1943, 15, 1-89. [CrossRef]

164. Lee, H.M. Evolution of galactic nuclei with 10-M_black holes. Mon. Not. R. Astron. Soc. 1995, 272, 605-617. [CrossRef]

165. Spitzer, L. Dynamical Evolution of Globular Clusters; Princeton University Press: Princeton, NJ, USA, 1987.

166. Gratton, R.G.; Fusi Pecci, F.; Carretta, E.; Clementini, G.; Corsi, C.E.; Lattanzi, M. Ages of Globular Clusters from HIPPARCOS Parallaxes of Local Subdwarfs. Astrophys. J. 1997, 491, 749-771. [CrossRef]

167. Gratton, R.G.; Bragaglia, A.; Carretta, E.; Clementini, G.; Desidera, S.; Grundahl, F.; Lucatello, S. Distances and ages of NGC 6397, NGC 6752 and 47 Tuc. Astron. Astrophys. 2003, 408, 529-543. [CrossRef]

168. VandenBerg, D.A.; Brogaard, K.; Leaman, R.; Casagrande, L. The Ages of 55 Globular Clusters as Determined Using an Improved Method along with Color-Magnitude Diagram Constraints, and Their Implications for Broader Issues. Astrophys. J. 2013, 775, 134. [CrossRef]

169. Tremaine, S.D.; Ostriker, J.P.; Spitzer, L.J. The formation of the nuclei of galaxies. I. M31. Astrophys. J. 1975, 196, 407-411. [CrossRef]

170. Capuzzo-Dolcetta, R. The Evolution of the Globular Cluster System in a Triaxial Galaxy: Can a Galactic Nucleus Form by Globular Cluster Capture? Astrophys. J. 1993, 415, 616. [CrossRef]

171. Capuzzo-Dolcetta, R.; Miocchi, P. Self-consistent simulations of nuclear cluster formation through globular cluster orbital decay and merging. Mon. Not. R. Astron. Soc. 2008, 388, L69-L73. [CrossRef]

172. Antonini, F.; Capuzzo-Dolcetta, R.; Mastrobuono-Battisti, A.; Merritt, D. Dissipationless Formation and Evolution of the Milky Way Nuclear Star Cluster. Astrophys. J. 2012, 750, 111. [CrossRef]

173. Antonini, F. Origin and Growth of Nuclear Star Clusters around Massive Black Holes. Astrophys. J. 2013, 763, 62. [CrossRef]

174. Arca-Sedda, M.; Capuzzo-Dolcetta, R. The globular cluster migratory origin of nuclear star clusters. Mon. Not. R. Astron. Soc. 2014, 444, 3738-3755. [CrossRef]

175. Arca-Sedda, M.; Capuzzo-Dolcetta, R.; Antonini, F.; Seth, A. Henize 2-10: The Ongoing Formation of a Nuclear Star Cluster around a Massive Black Hole. Astrophys. J. 2015, 806, 220. [CrossRef]

176. Mapelli, M.; Hayfield, T.; Mayer, L.; Wadsley, J. In Situ Formation of SgrA* Stars Via Disk Fragmentation: Parent Cloud Properties and Thermodynamics. Astrophys. J. 2012, 749, 168. [CrossRef]

177. Neumayer, N.; Seth, A.; Böker, T. Nuclear star clusters. Astron. Astrophys. Rev. 2020, 28, 4. [CrossRef]

178. Ade, P.A.R.; Aghanim, N.; Zonca, A.E.A. Planck 2015 results. XIII. Cosmological parameters. Astron. Astrophys. 2016, 594 , A13. [CrossRef]

179. Madau, P.; Fragos, T. Radiation Backgrounds at Cosmic Dawn: X-Rays from Compact Binaries. Astrophys. J. 2017, 840, 39. [CrossRef]

180. Santoliquido, F.; Mapelli, M.; Bouffanais, Y.; Giacobbo, N.; Di Carlo, U.N.; Rastello, S.; Artale, M.C.; Ballone, A. The cosmic merger rate density evolution of compact binaries formed in young star clusters and in isolated binaries. Astrophys. J. 2020, 898, 152. [CrossRef]

181. Ng, K.K.Y.; Vitale, S.; Farr, W.M.; Rodriguez, C.L. Probing Multiple Populations of Compact Binaries with Third-generation Gravitational-wave Detectors. Astrophys. J. Lett. 2021, 913, L5. [CrossRef]

182. Porciani, C.; Madau, P. On the Association of Gamma-Ray Bursts with Massive Stars: Implications for Number Counts and Lensing Statistics. Astrophys. J. 2001, 548, 522-531. [CrossRef] 
183. Palmerio, J.T.; Vergani, S.D.; Salvaterra, R.; Sanders, R.L.; Japelj, J.; Vidal-García, A.; D’Avanzo, P.; Corre, D.; Perley, D.A.; Shapley, A.E.; et al. Are long gamma-ray bursts biased tracers of star formation? Clues from the host galaxies of the Swift/BAT6 complete sample of bright LGRBs. III. Stellar masses, star formation rates, and metallicities at z > 1. Astron. Astrophys. 2019, 623, A26. [CrossRef]

184. Ferrarese, L.; Merritt, D. A Fundamental Relation between Supermassive Black Holes and Their Host Galaxies. Astrophys. J. Lett. 2000, 539, L9-L12. [CrossRef]

185. Gebhardt, K.; Bender, R.; Bower, G.; Dressler, A.; Faber, S.M.; Filippenko, A.V.; Green, R.; Grillmair, C.; Ho, L.C.; Kormendy, J.; et al. A Relationship between Nuclear Black Hole Mass and Galaxy Velocity Dispersion. Astrophys. J. Lett. 2000, 539, L13-L16. [CrossRef]

186. Graham, A.W.; Spitler, L.R. Quantifying the coexistence of massive black holes and dense nuclear star clusters. Mon. Not. R. Astron. Soc. 2009, 397, 2148-2162. [CrossRef]

187. Loredo, T.J. Accounting for source uncertainties in analyses of astronomical survey data. AIP Conf. Proc. 2004, 735, 195-206. [CrossRef]

188. Mandel, I.; Farr, W.M.; Gair, J.R. Extracting distribution parameters from multiple uncertain observations with selection biases. Mon. Not. R. Astron. Soc. 2019, 486, 1086-1093. [CrossRef]

189. Bouffanais, Y.; Mapelli, M.; Santoliquido, F.; Giacobbo, N.; Di Carlo, U.N.; Rastello, S.; Artale, M.C.; Iorio, G. New insights on binary black hole formation channels after GWTC-2: Young star clusters versus isolated binaries. Mon. Not. R. Astron. Soc. 2021, in press. [CrossRef]

190. Wong, K.W.K.; Breivik, K.; Kremer, K.; Callister, T. Joint constraints on the field-cluster mixing fraction, common envelope efficiency, and globular cluster radii from a population of binary hole mergers via deep learning. Phys. Rev. D 2021, $103,083021$. [CrossRef]

191. Zevin, M.; Bavera, S.S.; Berry, C.P.L.; Kalogera, V.; Fragos, T.; Marchant, P.; Rodriguez, C.L.; Antonini, F.; Holz, D.E.; Pankow, C. One Channel to Rule Them All? Constraining the Origins of Binary Black Holes Using Multiple Formation Pathways. Astrophys. J. 2021, 910, 152. [CrossRef]

192. Callister, T.A.; Farr, W.M.; Renzo, M. State of the field: Binary black hole natal kicks and prospects for isolated field formation after GWTC-2. arXiv 2020, arXiv:2011.09570.

193. Santoliquido, F.; Mapelli, M.; Giacobbo, N.; Bouffanais, Y.; Artale, M.C. The cosmic merger rate density of compact objects: Impact of star formation, metallicity, initial mass function, and binary evolution. Mon. Not. R. Astron. Soc. 2021, 502, 4877-4889. [CrossRef]

194. Broekgaarden, F.S.; Berger, E.; Neijssel, C.J.; Vigna-Gómez, A.; Chattopadhyay, D.; Stevenson, S.; Chruslinska, M.; Justham, S.; de Mink, S.E.; Mandel, I. Impact of Massive Binary Star and Cosmic Evolution on Gravitational Wave Observations I: Black HoleNeutron Star Mergers. arXiv 2021, arXiv:2103.02608.

195. Breen, P.G.; Heggie, D.C. Dynamical evolution of black hole subsystems in idealized star clusters. Mon. Not. R. Astron. Soc. 2013, 432, 2779-2797. [CrossRef]

196. Breen, P.G.; Heggie, D.C. On black hole subsystems in idealized nuclear star clusters. Mon. Not. R. Astron. Soc. 2013, 436, 584-589. [CrossRef]

197. Morscher, M.; Pattabiraman, B.; Rodriguez, C.; Rasio, F.A.; Umbreit, S. The Dynamical Evolution of Stellar Black Holes in Globular Clusters. Astrophys. J. 2015, 800, 9. [CrossRef]

198. Wang, L. The survival of star clusters with black hole subsystems. Mon. Not. R. Astron. Soc. 2020, 491, 2413-2423. [CrossRef]

199. Hénon, M. Sur l'évolution dynamique des amas globulaires. II. Amas isol\&eacute. Ann. D'Astrophys. 1965, $28,62$.

200. Yang, Y.; Bartos, I.; Haiman, Z.; Kocsis, B.; Márka, S.; Tagawa, H. Cosmic Evolution of Stellar-mass Black Hole Merger Rate in Active Galactic Nuclei. arXiv 2020, arXiv:2003.08564. 